\title{
A Practical Approach to 3D Scanning in the Presence of Interreflections, Subsurface Scattering and Defocus
}

\author{
Mohit Gupta - Amit Agrawal - Ashok Veeraraghavan - Srinivasa G. \\ Narasimhan
}

Received: date / Accepted: date

\begin{abstract}
Global or indirect illumination effects such as interreflections and subsurface scattering severely degrade the performance of structured light-based 3D scanning. In this paper, we analyze the errors in structured light, caused by both long-range (interreflections) and short-range (subsurface scattering) indirect illumination. The errors depend on the frequency of the projected patterns, and the nature of indirect illumination. In particular, we show that long-range effects cause decoding errors for low-frequency patterns, whereas shortrange effects affect high-frequency patterns.
\end{abstract}

Based on this analysis, we present a practical 3D scanning system which works in the presence of a broad range of indirect illumination. First, we design binary structured light patterns that are resilient to individual indirect illumination effects using simple logical operations and tools from combinatorial mathematics. Scenes exhibiting multiple phenomena are handled by combining results from a small ensemble of such patterns. This combination also allows detecting any residual errors that are corrected by acquiring a few additional images. Our methods can be readily incorporated into existing scanning systems without significant overhead in terms

\footnotetext{
A preliminary version of this paper appeared in [16]. Mohit Gupta is with the Computer Science Department, Columbia University, New York, NY, 10027.

E-mail: mohitg@cs.columbia.edu

Amit Agrawal is with the Mitsubishi Electrical Research Labs, Cambridge, MA, 02139.

E-mail: agrawal@merl.com

Ashok Veeraraghavan is with the Electrical and Computer Engineering Department, Rice University, Houston, TX, 77005.

E-mail:vashok@rice.edu

Srinivasa G. Narasimhan is with the Robotics Institute, Carnegie Mellon University, Pittsburgh, PA, 15213.

E-mail: srinivas@cs.cmu.edu
}

of capture time or hardware. We show results for several scenes with complex shape and material properties.

Keywords Structured light 3D scanning, interreflections, subsurface scattering, defocus, global illumination, indirect illumination, light transport, projectors.

\section{Introduction}

Structured light triangulation has become the method of choice for shape measurement in several applications including industrial automation, graphics, humancomputer interaction and surgery. Since the early work in the field about 40 years ago $[37,25,33]$, research has been driven by two factors: reducing the acquisition time and increasing the depth resolution. Significant progress has been made on both fronts (see the survey by Salvi et al. [34]) as demonstrated by systems which can recover shapes at close to $1000 \mathrm{~Hz}$. [41] and at a depth resolution better than 30 microns [11].

Despite these advances, the applicability of most structured light techniques remains limited to well behaved scenes. It is assumed that scene points receive illumination only directly from the light source. For many real world scenarios, this is not true. Imagine a robot trying to navigate an underground cave or an indoor scenario, a surgical instrument inside human body, a robotic arm sorting a heap of metallic machine parts, or a movie director wanting to image the face of an actor. In all these settings, scene points receive illumination indirectly in the form of interreflections, subsurface or volumetric scattering. Such effects, col- 


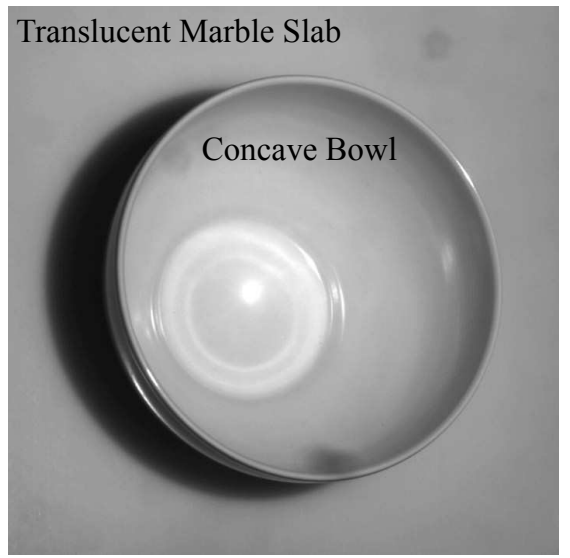

(a) Concave bowl on a translucent marble slab

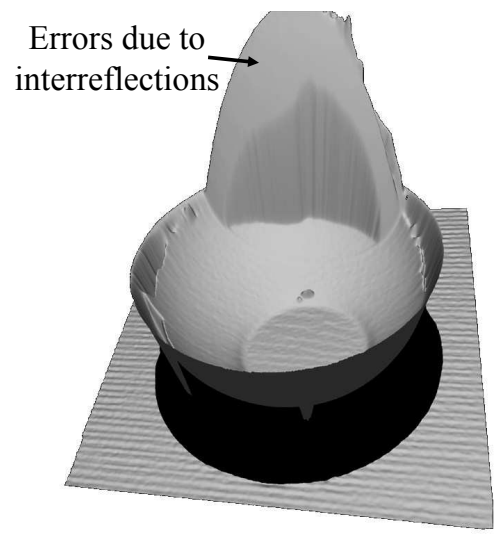

(d) Shape recovered using Conventional Gray codes (11 images)

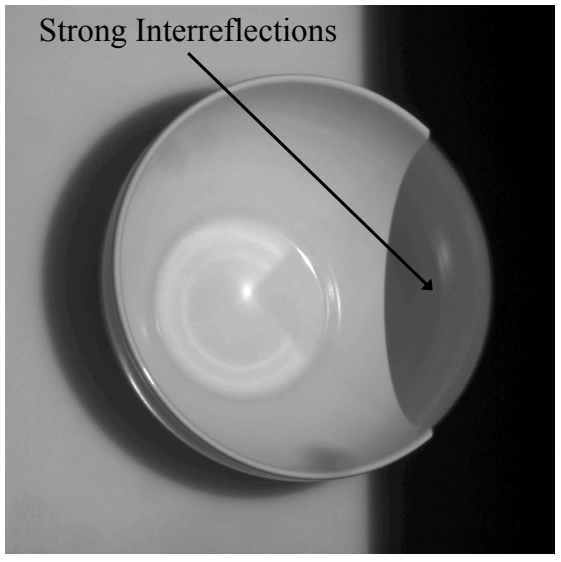

(b) Input image under low-frequency illumination

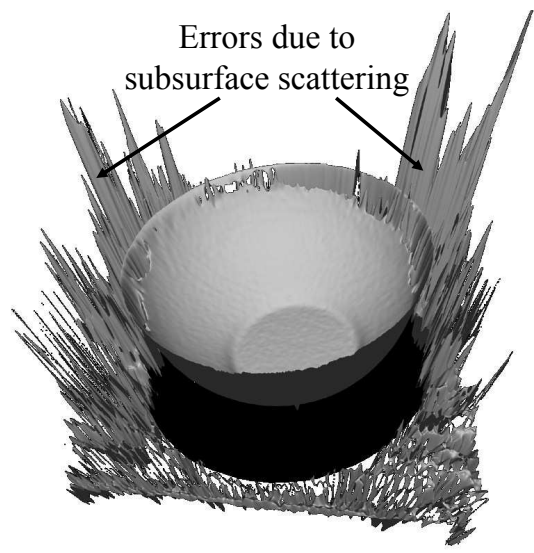

(e) Shape recovered using Modulated phase shifting [8] (162 images)

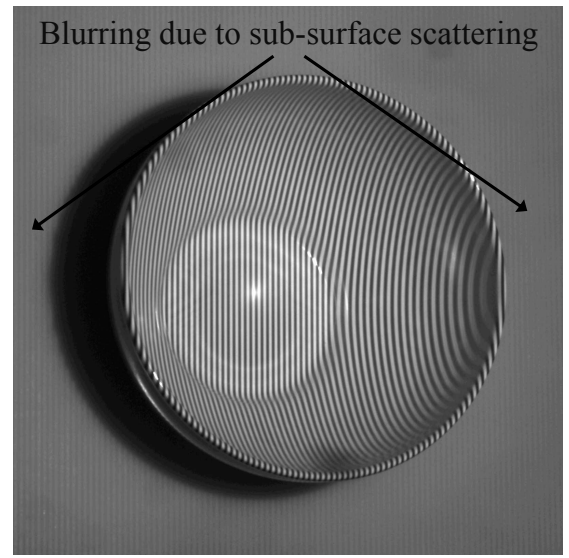

(c) Input image under high-frequency illumination

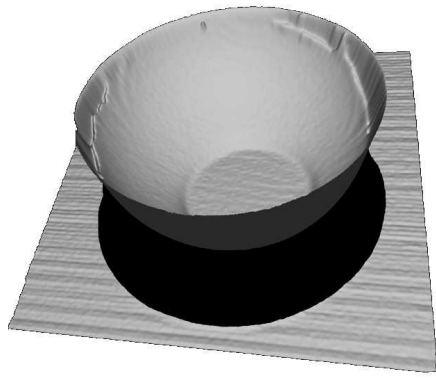

(f) Shape recovered using our ensemble codes (42 images)

Fig. 1 Measuring shape for the 'bowl on marble-slab' scene. This scene is challenging because of strong interreflections inside the concave bowl and subsurface scattering on the translucent marble slab. (b) Scene points inside the bowl which are not directly illuminated receive substantial irradiance due to interreflections. (d) This results in systematic errors in the recovered depth. (c) Due to subsurface scattering on the translucent marble slab, high-frequency illumination patterns are severely blurred. Notice the low contrast of the stripes as compared to the bowl. (e) This results in depth-errors on the marble-slab. (f) Our technique uses an ensemble of codes optimized for individual indirect illumination effects, and results in an accurate shape reconstruction. Parentheses contain the number of input images. More results and comparisons with existing techniques are at the project web-page [1].

lectively termed global or indirect illumination ${ }^{1}$, often dominate the direct illumination and strongly depend on the shape and material properties of the scene. Not accounting for these effects results in large errors in the recovered shape (see Figure 1b). Because of the systematic nature of these errors ${ }^{2}$, it is hard to correct them in post-processing.

The goal of this paper is to build an end-to-end system for structured light 3D scanning under a broad

\footnotetext{
${ }^{1}$ Global illumination should not be confused with the oft-used "ambient illumination" that is subtracted by capturing image with the structured light source turned off.

${ }^{2}$ In photometric stereo, interreflections result in a shallow but smooth reconstruction [29,28]. In structured light 3D scanning, interreflections result in local errors.
}

range of indirect illumination effects. The focus is on designing the projected patterns (coding) and decoding schemes. In particular, we consider binary structured light patterns, which are perhaps the simplest to implement and widely used in several research and commercial systems. The key observation is that different indirect illumination effects place contrasting constraints on the spatial frequencies of projected structured light patterns. In particular, interreflections result in errors for low-frequency structured light patterns ${ }^{3}$. On the

3 Strictly speaking, since all binary patterns have step edges, all of them have high spatial frequencies. For the analysis and discussion in this paper, low-frequency patterns implies patterns with thick stripes. Similarly, high-frequency patterns mean patterns with only thin stripes. 
other hand, local effects such as subsurface scattering and defocus blur the high-frequency patterns, making it hard to decode them reliably.

We design patterns that modulate indirect illumination and prevent the errors at capture time itself. We show that it is possible to construct codes with only high-frequency binary patterns by introducing the concept of logical coding and decoding. The key idea is to express low-frequency patterns as pixel-wise logical combinations of two high-frequency patterns. Because of high frequencies, these patterns are resilient to longrange effects. In order to deal with short-range effects, we use tools from combinatorial mathematics to design patterns consisting solely of low frequencies. In comparison, most currently used patterns (e.g., Gray codes) contain a combination of both low and high spatial frequencies, and thus are ill-equipped to deal with indirect illumination.

Indirect illumination in most real world scenes is not limited to either short or long-range effects. Codes optimized for long-range effects make errors in the presence of short-range effects and vice versa. How do we handle scenes that exhibit more than one type of indirect illumination effect (such as the one in Figure 1(a))? To answer this, we observe that the probability of two different codes producing the same erroneous decoding is very low. This observation allows us to project a small ensemble of codes and use a simple voting scheme to compute the correct decoding at every pixel, without any prior knowledge about the scene (Figure 1(d)).

Finally, for highly challenging scenes, we present an error detection scheme based on a simple consistency check over the results of the individual codes in the ensemble. We then use an error correction scheme which further reduces the errors due to indirect illumination by selectively re-illuminating only the incorrectly reconstructed scene points [38]. We demonstrate accurate reconstructions on scenes with complex geometry and material properties, such as shiny brushed metal, translucent wax and marble and thick plastic diffusers (like shower curtains). Our techniques outperform many existing schemes while using significantly fewer images (12-42 versus 200-700) as compared to previous work dealing with indirect illumination. We believe that these techniques are important steps towards making 3D scanning techniques applicable to a large class of complex, real world scenarios.

\section{Related Work}

Structured light 3D Scanning: 3D scanning using structured light is one of the oldest computer vision techniques. Since the first papers $[37,25,33]$, a lot of progress has been made in terms of reconstruction speed, accuracy and resolution. Broadly, these techniques are divided into discrete [22] and continuous [40] coding schemes. For an exhaustive survey on structured light techniques, reader is referred to the survey by Salvi et al [34]. In addition, hybrid techniques that combine structured light with photometric stereo based techniques have been proposed as well $[30,3]$.

Shape recovery in the presence of indirect illumination: The seminal work of Nayar et al. [28] presented an iterative approach for reconstructing shape of Lambertian objects in the presence of interreflections. Liu et al. [24] proposed a method to estimate the geometry of a Lambertian scene by using the second bounce light transport matrix. Gupta et al. [19] presented methods for recovering depths using projector defocus [39] under indirect illumination effects. Chandraker et al. [6] use interreflections to resolve the basrelief ambiguity inherent in shape-from-shading techniques. Holroyd et al. [21] proposed an active multiview stereo technique where high-frequency illumination is used as scene texture that is invariant to indirect illumination. Park et al. $[32,31]$ move the camera or the scene to mitigate the errors due to indirect illumination in a structured light setup. Hermans et al. [20] use a moving projector in a variant of structured light triangulation. The depth measure used in this technique (frequency of the intensity profile at each pixel) is invariant to indirect illumination. In this paper, our focus is on designing structured light systems that are applicable for a wide range of scenes, and which require a single camera and a projector, without any moving parts.

Nayar et al. showed that the direct and indirect components of scene radiance could be efficiently separated [29] using high-frequency illumination patterns. This has led to several attempts to perform structured light scanning under indirect illumination $[7,8,14]$. All these techniques rely on subtracting or reducing the indirect component and apply conventional approaches on the residual direct component. While these approaches have shown promise, there are three issues that prevent them from being applicable broadly: (a) the direct component estimation may fail due to strong interreflections (as with shiny metallic parts), (b) the residual direct component may be too low and noisy (as with translucent surfaces, milk and murky water), and (c) they require significantly higher number of images than traditional approaches, or rely on weak cues like polarization. Recently, Couture et al. [9] proposed using band-pass unstructured patterns to handle interreflections. Their approach involves capturing a large 
number (200) of images with random high-frequency patterns projected on the scene. In contrast, we explicitly design ensembles of illumination patterns that are resilient to a broader range of indirect illumination effects (interreflections, subsurface scattering, defocus, diffusion, and combinations of multiple effects), while using significantly fewer images.

Shape recovery in other optically challenging scenarios: Active illumination has also been used to measure density distribution of volumetric media [4, $15]$ and reconstruct transparent objects $[35,26]$. For a detailed survey on techniques for reconstructing transparent and specular surfaces, please refer to the state of the art report by Ihrke et al. [23]. There have also been techniques for performing $3 \mathrm{D}$ scanning in the presence of volumetric media using light striping $[27,17]$. Our techniques can not handle volumetric scattering. The focus of this work is on reconstructing opaque and translucent surfaces with complex shapes.

\section{Analysis of Errors due to Indirect Illumination}

In this section, we analyze errors in structured light caused due to different indirect illumination effects. The basic principle behind shape from structured light is triangulation. Each projector row/column is encoded with a unique spatial or temporal code. A projector illuminates the scene with the assigned code and camera takes a sequence of images, one for each projected pattern. For each camera pixel, the corresponding projector row/column is found by decoding the measured intensity values. Once the correspondence is computed, depth is computed by triangulation.

The resulting depth estimate is incorrect if there is an error in estimating the correspondence. The magnitude of errors depends on the region of influence of indirect illumination at any scene point. For instance, some scene points may receive indirect illumination only from a local neighborhood (subsurface scattering). We call these short-range effects. Some points may receive indirect illumination from a larger region (interreflections or diffusion). We call these long-range effects. As shown in Figures 2 and 3, long-range effects and short range effects result in incorrect decoding of low and high spatial frequency patterns, respectively. We analyze these errors for the case of binary structured light patterns.

Binary patterns are decoded by binarizing the captured images into illuminated vs. non-illuminated pixels. A robust way to do this is to capture two images $L$ and $\bar{L}$, under the pattern $P$ and the inverse pattern
$\bar{P}$, respectively ${ }^{4}$. For a scene point $S^{i}$, its irradiances $L^{i}$ and $\overline{L^{i}}$ are compared. If, $L^{i}>\overline{L^{i}}$, then the point is classified as directly lit. A fundamental assumption for correct binarization is that each scene point receives irradiance from only a single illumination element (light stripe or a projector pixel). However, due to indirect illumination effects and projector defocus, a scene point can receive irradiance from multiple projector pixels, resulting in incorrect binarization.

In the following, we derive the condition for correct binarization in the presence of indirect illumination and defocus. Suppose $S^{i}$ is directly lit under a pattern $P$. The irradiances $L^{i}$ and $\overline{L^{i}}$ are given as:

$L^{i}=L_{d}^{i}+\beta L_{g}^{i}$,

$\overline{L^{i}}=(1-\beta) L_{g}^{i}$,

where $L_{d}^{i}$ and $L_{g}^{i}$ are the direct and indirect components of the irradiance at $S^{i}$ [29], when the scene is fully lit. $\beta$ is the fraction of the indirect component under the pattern $P$.

In the presence of defocus (projector or camera), the projected patterns and the captured image is blurred. Similarly, aberrations due to imperfect projector optics also result in blurring of the projected patterns. The blur influences the highest frequency patterns, often completely blurring them out ${ }^{5}$. Defocus, unlike indirect illumination effects, modulates the direct component as well, as shown in [19]:

$L^{i}=\alpha L_{d}^{i}+\beta L_{g}^{i}$

$\overline{L^{i}}=(1-\alpha) L_{d}^{i}+(1-\beta) L_{g}^{i}$.

The fractions $(\alpha$ and $1-\alpha)$ depend on the projected pattern and the amount of defocus. In the absence of defocus, $\alpha=1$. For correct binarization, it is required that $L_{i}>\overline{L_{i}}$, i.e.

$\alpha L_{d}^{i}+\beta L_{g}^{i}>(1-\alpha) L_{d}^{i}+(1-\beta) L_{g}^{i}$

This condition is satisfied in the absence of indirect illumination $\left(L_{g}^{i}=0\right)$ and defocus $(\alpha=1)$. Next, we analyze the errors in the binarization process due to different indirect illumination effects and defocus ${ }^{6}$.

\footnotetext{
4 The inverse pattern can be generated by subtracting the image from image of the fully lit scene.

${ }^{5}$ For example, pico-projectors are increasingly getting popular for structured light applications in industrial assembly lines. However, due to imperfect optics, they can not resolve patterns with thin stripes, for example, a striped pattern of 2-pixel width.

${ }^{6}$ Errors for the particular case of laser range scanning of translucent materials were analyzed in [13]. Errors due to sensor noise and spatial mis-alignment of projector-camera pixels were analyzed in [36].
} 


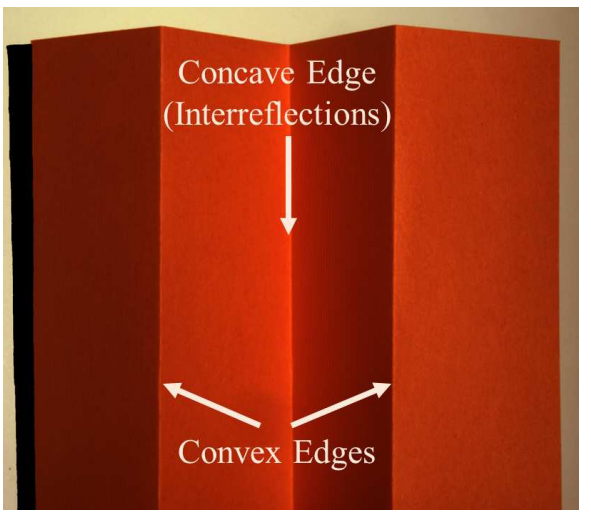

Scene with interreflections: Concave V-groove

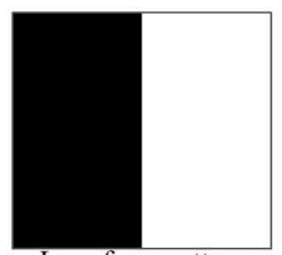

Low freq. pattern

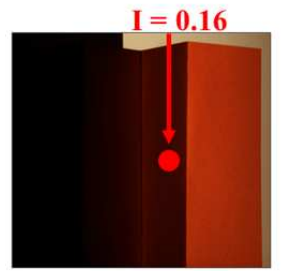

\section{Captured Images}

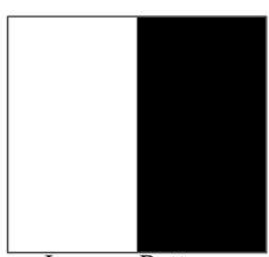

Inverse Pattern $\overline{\mathrm{I}}=0.25$

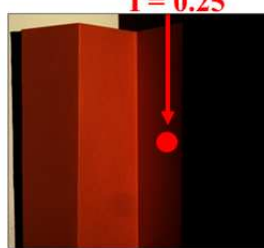

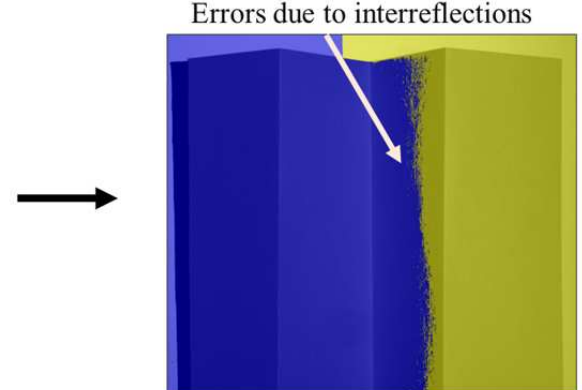

Binarization

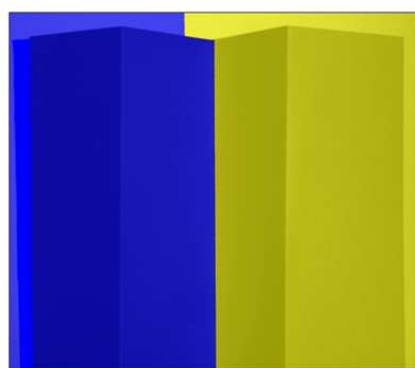

Ground Truth Binarization

One (illuminated)

Zero (not-illuminated)

Illustration of errors due to interreflections (color online): The scene point marked with red is directly illuminated when the low-frequency pattern is projected on the scene. It is not directly lit when the inverse pattern is projected. Due to interreflections, its intensity is higher when it is not directly lit (0.25) as compared to when it is directly lit (0.16). This results in a decoding (binarization) error, as shown on the right. Scene points decoded as one (directly illuminated) are marked in yellow and points decoded as zero (not illuminated) are marked in blue. In the correct decoding, only the points to the left of the concave edge should be zero.

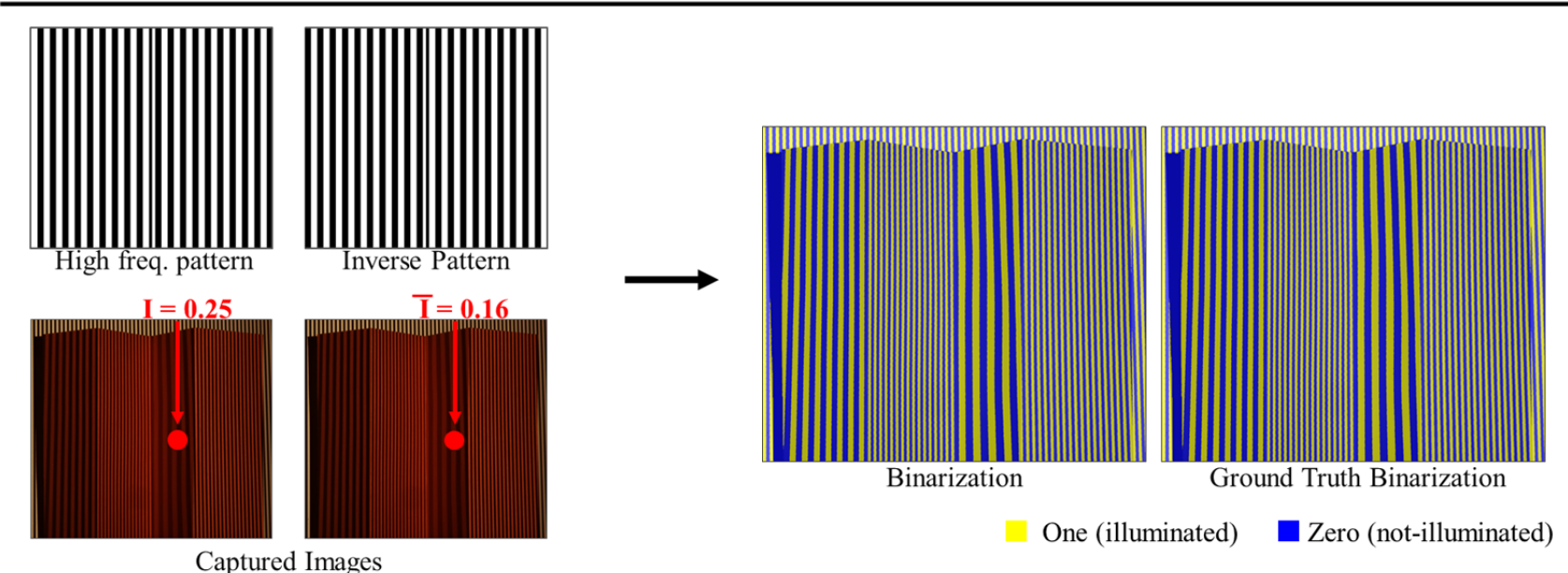

Decoding for high-frequency patterns: High-frequency patterns are decoded correctly even in the presence of interreflections. See Section 3.1 for a detailed explanation.

Fig. 2 Structured light decoding in the presence of interreflections (color online). Top: A concave v-groove. Middle: Illustration of structured light decoding errors due to interreflections. Interreflections result in low-frequency patterns being decoded incorrectly. Bottom High-frequency patterns are decoded correctly. 


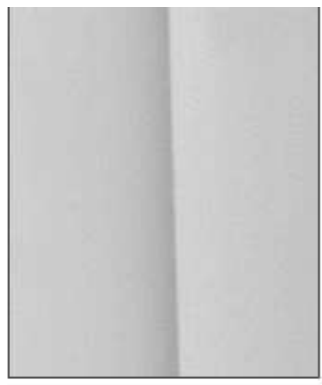

(a)

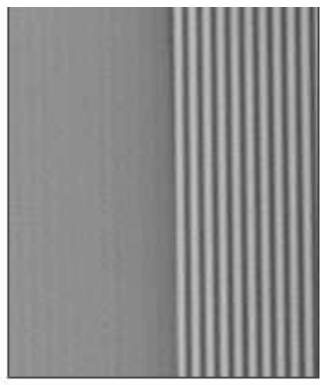

(b)

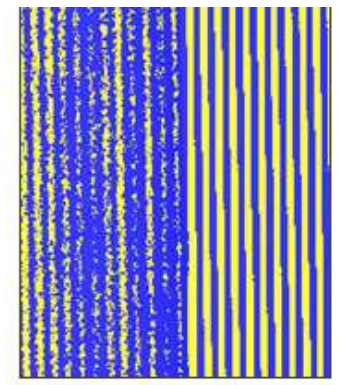

(c)

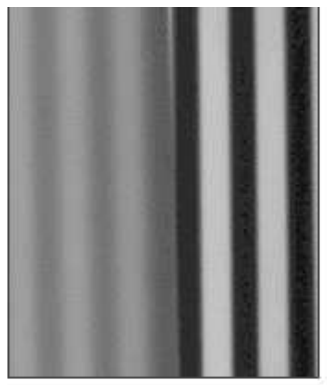

(d)

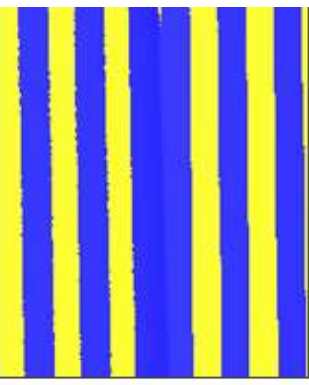

(e)

Fig. 3 Structured light decoding in the presence of subsurface scattering: (a) This scene consists of a translucent slab of marble on the left and an opaque plane on the right. (b) A high-frequency pattern is severely blurred on the marble. Consequently, binarization information can not be extracted reliably on the marble slab (c). In contrast, the image captured (d) under a low-frequency pattern is binarized (e) more accurately.

\subsection{Long-range effects (interreflections)}

Suppose a scene point $S^{i}$ receives most of the indirect component when it is not directly lit $(\beta \approx 0$ in Eqs. 1 4 ), and the indirect component is larger than the direct component $\left(L_{d}^{i}<L_{g}^{i}\right)$. Substituting in the binarization condition (Eq. 5), we get $L^{i}<\overline{L^{i}}$, which results in a binarization error. Such a situation may arise due to longrange interreflections, when scenes are illuminated with low-frequency patterns. This is because low-frequency patterns illuminate the scene asymmetrically. For example, consider the v-groove concavity shown in Figure 2. Under a low-frequency pattern, several points in the concavity are brighter when they are not directly lit, resulting in a binarization error.

On the other hand, if the scene is illuminated with a high-frequency pattern, the captured image is binarized correctly even in the presence of interreflections. This is explained as follows. If a high-frequency pattern (with equal off and on pixels) is projected on the scene, scene points receive approximately half the indirect component, i.e., $\beta \approx 0.5$ [29]. Thus, for a scene point $S^{i}, L^{i} \approx L_{d}^{i}+0.5 L_{g}^{i}$ and $\overline{L^{i}} \approx 0.5 L_{g}^{i}$. Consequently, $L^{i} \geq \overline{L^{i}}$, and the condition for correct binarization is satisfied. An example is shown in Figure 2.

\subsection{Short-range effects (subsurface scattering and defocus)}

Short-range effects result in low-pass filtering of the incident illumination. In the context of structured light, these effects may severely blur the high-frequency patterns, making it hard to correctly binarize them. This can be explained in terms of the binarization condition in Eq. 5. For high-frequency patterns, $\beta \approx 0.5$ [29]. If the difference in the direct terms $\left|\alpha L_{d}^{i}-(1-\alpha) L_{d}^{i}\right|$ is small, either because the direct component is low due to subsurface scattering $\left(L_{d}^{i} \approx 0\right)$ or because of severe defocus $(\alpha \approx 0.5)$, the pattern can not be binarized robustly. An example is shown in Figure 3.

For short-range effects, most of the indirect illumination at a scene point comes from a local neighborhood. Suppose a low-frequency patterns is projected on the scene. If a scene point is directly illuminated, most of its local neighborhood is directly illuminated as well. Hence, $\alpha \geq 0.5$ and $\beta \geq 0.5$. Thus, for low-frequency patterns, short-range effects actually help in correct decoding even when the direct component is low.

For conventional Gray codes, the high-frequency patterns correspond to the lower significance bits. Incorrect decoding of high-frequency patterns results in a loss of depth resolution. For example, when conventional Gray codes are used, if patterns of stripe-width less than 5 pixels are not resolved, last 2 bits of information are lost. An example is shown in Figure 6.

In summary, long and short-range effects respond differently to the spatial frequencies of the incident illumination. In the presence of longrange effects, low-frequency patterns are susceptible to incorrect binarization, whereas high-frequency patterns are decoded correctly. On the other hand, for shortrange effects, high-frequency patterns are susceptible to coding errors while the low-frequency patterns are decoded accurately.

\section{Patterns for Error Prevention}

In this section, we design patterns that modulate indirect illumination and prevent errors at capture time itself. Because of the contrasting requirements on spatial frequencies (as discussed in the previous section), it is clear that we need different codes for different indirect illumination effects. For long-range effects, we want patterns with only high frequencies (low maximum stripe- 
widths). For short-range effects, we want patterns with only low frequencies (high minimum stripe-widths). However, most currently used patterns contain a combination of both low and high spatial frequencies. How do we design patterns with only low or only high frequencies? We show that by performing simple logical operations, it is possible to design codes with only high frequency patterns. For short-range effects, we draw on tools from the combinatorial maths literature to design binary codes with large minimum stripe-widths, resulting in patterns with low spatial frequencies.

\subsection{Logical coding-decoding for long-range effects}

We introduce the concept of logical coding and decoding to design patterns with only high frequencies. An example is given in Figure 4. For binary structured light, the goal is to correctly binarize the captured images. We model the binarization process as a function from the set of binary projected patterns $(\mathbb{P})$ to the set of binary classifications of the captured image $(\mathbb{B})$ :

$f: \mathbb{P} \Rightarrow \mathbb{B}$.

For a pattern $P \in \mathbb{P}, f(P)$ is the binarization of the captured image if the scene is illuminated by $P$. As discussed in the previous section, under interreflections, $f(P)$ is computed robustly if $P$ is a high-frequency pattern. But, if $P$ is a low-frequency pattern, $f(P)$ may be computed incorrectly. How do we ensure that $f(P)$ is computed correctly even for low-frequency patterns? We propose decomposing a low-frequency pattern $P_{l f}$ into two high-frequency patterns $P_{h f}^{1}$ and $P_{h f}^{2}$ using pixel-wise binary operators $\odot$ and $\widehat{\odot}$, such that:

$f\left(P_{l f}\right)=f\left(P_{h f}^{1} \odot P_{h f}^{2}\right)=f\left(P_{h f}^{1}\right) \widehat{\odot} f\left(P_{h f}^{2}\right)$

If we find such a decomposition, we can robustly compute the binarizations $f\left(P_{h f}^{1}\right)$ and $f\left(P_{h f}^{2}\right)$, and combine these to achieve the correct binarization $f\left(P_{l f}\right)$. Two questions remain: (a) What binary operators can be used? (b) How can we decompose a low frequency pattern into two high-frequency patterns? For both binary operators, we choose the logical XOR $(\otimes)$ because it has the following properties. First, the binarization function $f$ is distributive with respect to XOR:

$f\left(P_{h f}^{1} \otimes P_{h f}^{2}\right)=f\left(P_{h f}^{1}\right) \otimes f\left(P_{h f}^{2}\right)$

This property allows decomposing a pattern into two patterns, then computing and combining their binarizations to achieve the binarization for the original pattern. Second, the all zero pattern $\mathbf{0}$ is the identity for XOR, i.e., $P \otimes \mathbf{0}=P$. Third, XORing a pattern with itself gives the zero pattern, i.e., $P \otimes P=\mathbf{0}$. Fourth, $\mathrm{XOR}$ is associative, i.e., for any three patterns $P, Q, R$, $(P \otimes Q) \otimes R=P \otimes(Q \otimes R)$. Using these three properties, it is easy to show that if $P_{l f}=P_{h f}^{1} \otimes P_{h f}^{2}$, then $P_{h f}^{2}=P_{l f} \otimes P_{h f}^{1}$.

This provides a simple means to find the decomposition for a low-frequency pattern $P_{l f}$. First, choose a high-frequency pattern $P_{h f}^{1}$. The second pattern $P_{h f}^{2}$ is then computed by simply taking the pixel-wise logical XOR of $P_{l f}$ and $P_{h f}^{1}$. We call the first high-frequency pattern the base pattern. Instead of the low-frequency pattern, the two high-frequency patterns $P_{h f}^{1}$ and $P_{h f}^{2}$ are projected on the scene. The corresponding captured images are binarized. The two binarizations are then combined by performing another pixel-wise logical XOR operation. This produces the correct binarization as if the scene was illuminated by the original low-frequency pattern. An example is shown in Figure 4.

The logical patterns can be constructed by taking the pixel-wise logical XOR of a high-frequency pattern (base pattern) in the conventional Gray codes with all other patterns. This is illustrated in Figure 4. The resulting patterns have only high spatial frequencies. Note that there is no overhead introduced; the number of projected patterns remains the same as the conventional codes. If the last Gray code pattern is chosen as the base plane, the resulting codes are called logical XOR02 codes. All the projected patterns have a maximum stripe width of 2 pixels. In contrast, the original Gray codes have a maximum stripe-width of 512 pixels.

If the second-to-last pattern is used as the base plane, the resulting codes are called logical XOR-04 codes. The last pattern is projected unmodified. In these codes, all the projected patterns have a maximum stripewidth of 4 pixels. In general, if the $(n-k)^{t h}$ pattern is used as the base plane, the resulting codes are called logical XOR- $2^{k+1}$ codes, where $n$ is the total number of projected patterns. The maximum stripe width is $2^{k+1}$ pixels and the last $k-1$ planes are projected unmodified. The patterns for logical XOR-02 and XOR-04 codes are shown in Figure 7 . The pattern images can be downloaded from the project web-page [1].

Color Logical XOR Codes: Color can be used to reduce the number of required input images ${ }^{7}$ as compared to binary patterns. It is possible to construct

7 The color of the incident illumination can be decoded from the image of the illuminated scenes on a per-pixel basis, even for non-white scenes [5]. It is not required to assume spatial smoothness or color neutrality of the scene. 


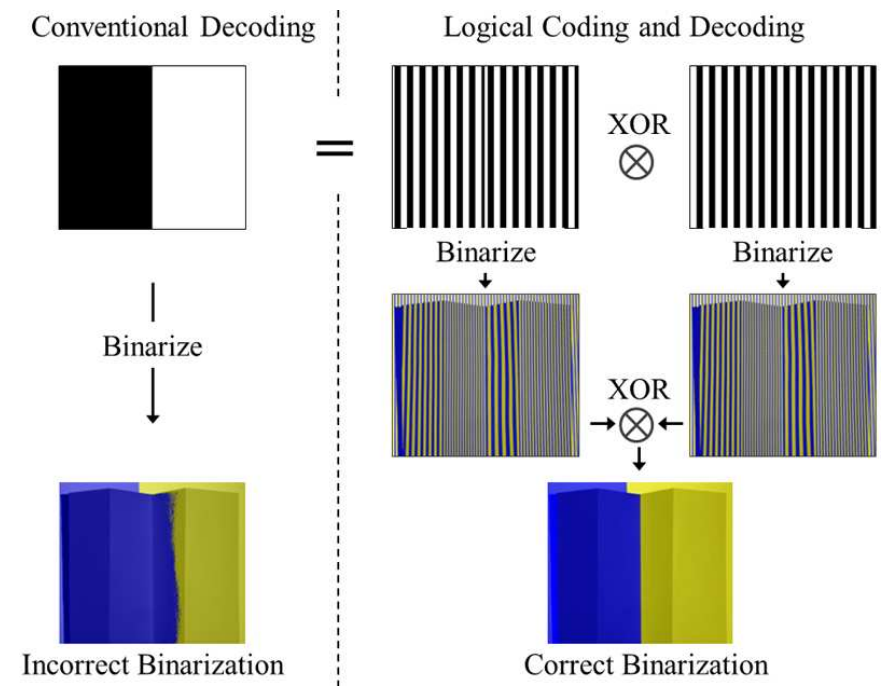

(a) Conventional Decoding vs. Logical Coding and Decoding

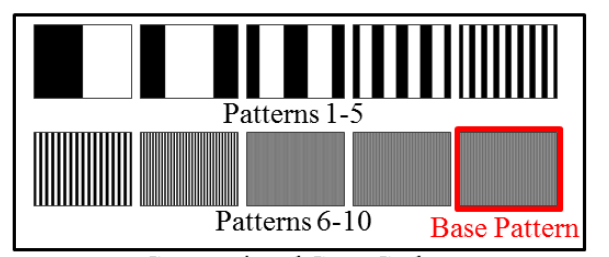

Conventional Gray Codes

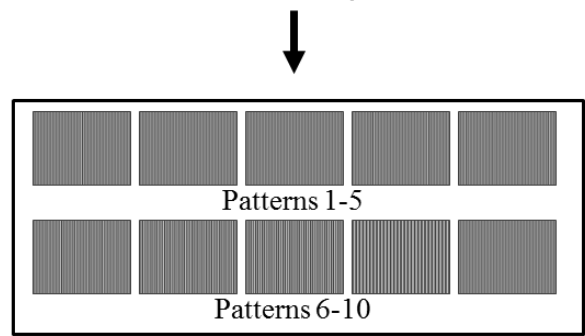

High spatial frequency XOR-02 Codes

(b) Generation of Logical XOR Patterns

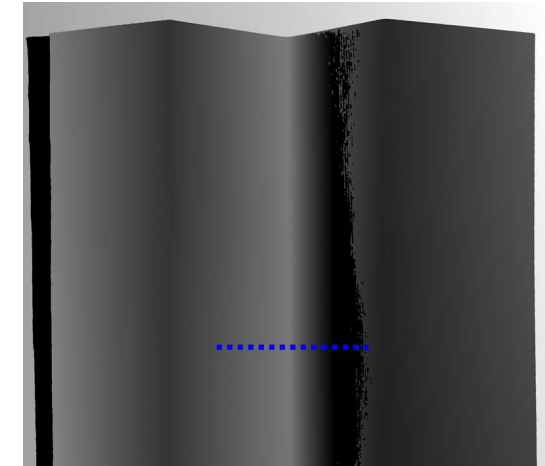

(c) Depth (conventional Gray codes) Mean absolute error $=28.8 \mathrm{~mm}$

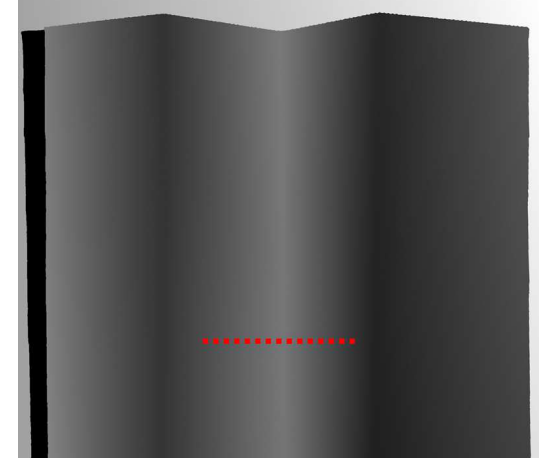

(d) Depth (our Logical XOR04 codes) Mean absolute error $=1.4 \mathrm{~mm}$

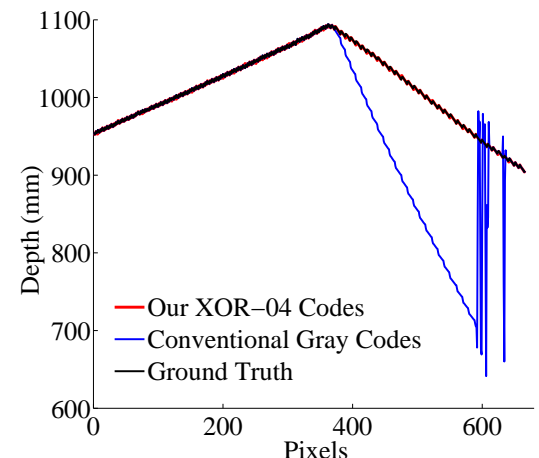

(e) Comparison with the ground truth

Fig. 4 Logical coding and decoding for long range indirect illumination (color online): (a) In logical coding and decoding, a low-frequency pattern is expressed as a pixel-wise logical combination (e.g., XOR) of two high-frequency patterns. The high-frequency patterns are projected on the scene and the captured images are binarized. The two binarizations are combined by performing another pixel-wise XOR operation. This produces the correct binarization as if the scene was illuminated by the original low-frequency pattern. (b) The logical patterns can be constructed by taking the pixel-wise logical XOR of a high-frequency pattern (base plane) in the conventional Gray codes with all other patterns. The resulting patterns have only high spatial frequencies. The number of projected images remains the same. If the last pattern is chosen as the base plane, the resulting codes are called logical XOR-02 codes. If the second-to-last pattern is used as the base plane, the resulting codes are called logical XOR-04 codes. (c) Depth map computed with the conventional codes. Because of incorrect binarization of the low-frequency patterns, depth map has large and systematic errors. Because of their systematic nature, these cannot be removed by simple smoothing in post-processing. (d) Depth map computed using our logical XOR-04 codes. The errors due to interreflections have been significantly reduced. (e) Comparison with the ground-truth.

color logical XOR codes by performing logical operations, similar to the binary case.

We consider the case where each color channel at a projector pixel has a binary value. Thus, each projector pixel can take 8 possible color values - $\{$ RGB $\}$ $=\{000,001,010,011,100,101,110,111\}$. For example, if a projector pixel is encoded as $\{100\}$, its red channel is 1 , and the green and blue channels are 0 . In this case, $N_{\text {color }}=\left\lceil\log _{8}(M)\right\rceil$ patterns are required to uniquely encode $M$ projector columns. In contrast, for binary coding schemes, $N_{\text {bin }}=\left\lceil\log _{2}(M)\right\rceil$ patterns are required to encode $M$ different projector columns. For example, if $M=512, N_{\text {color }}=3$ and $N_{\text {bin }}=9$.

Figure 5 (a-c) shows color Gray codes for a projector with 512 columns. These codes were generated using the $\mathrm{K}$-ary $(K=8)$ reflected Gray code construction [10]. Figures 5 (d-f) show input images of a concave bowl under the color Gray codes. Due to low frequencies in the projected patterns, interreflections result in er- 


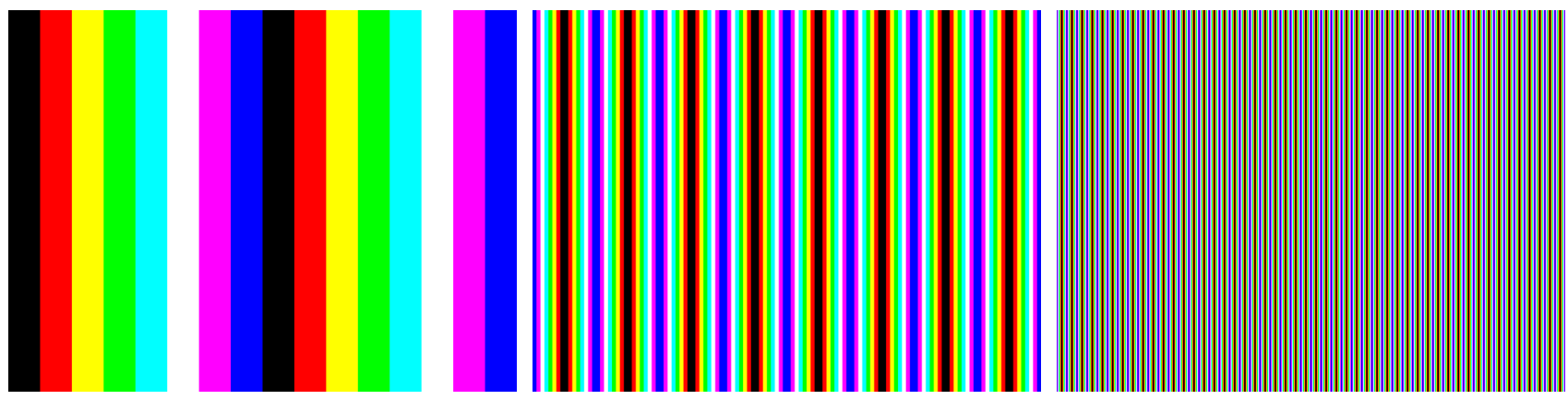

(a-c) Color Gray codes

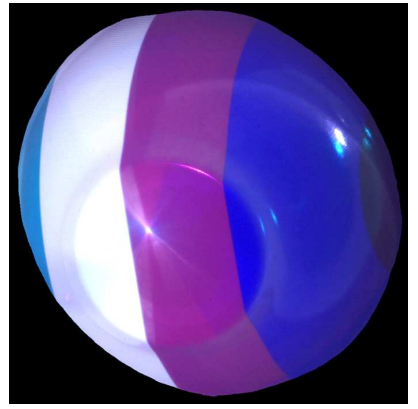

(d-f) Input images for concave bowl using color Gray codes
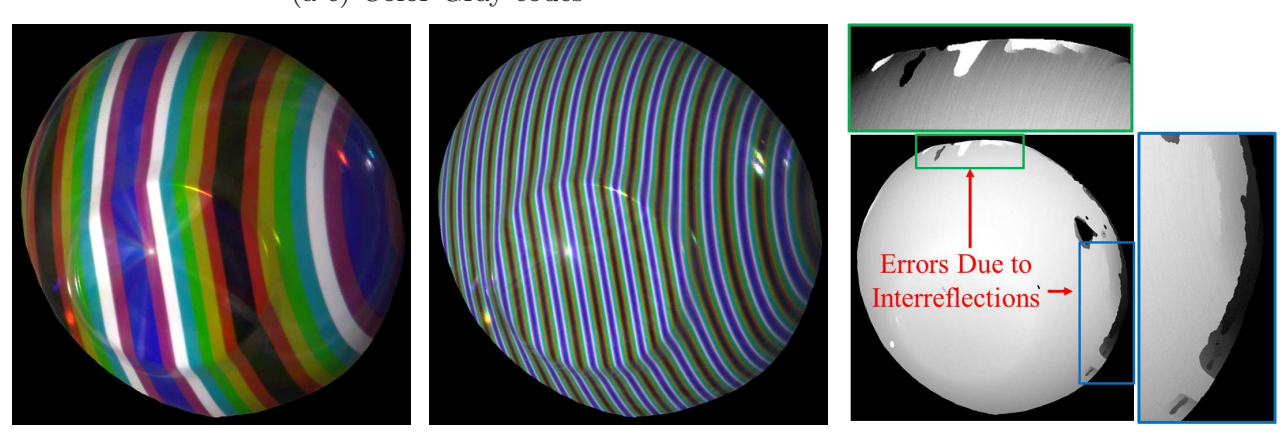

(g) Depth map
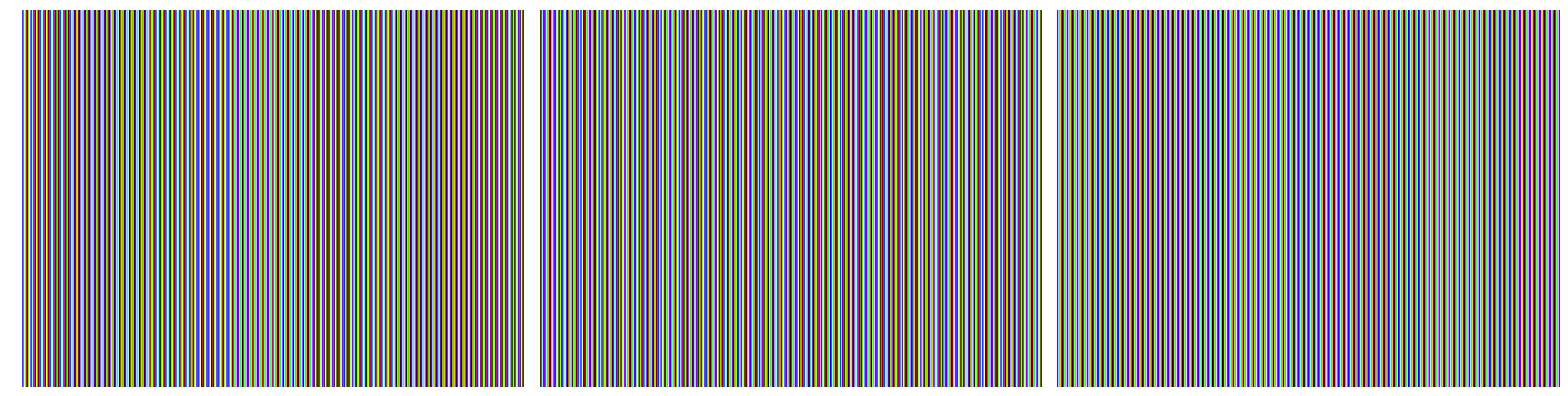

(h-j) Color Logical XOR codes

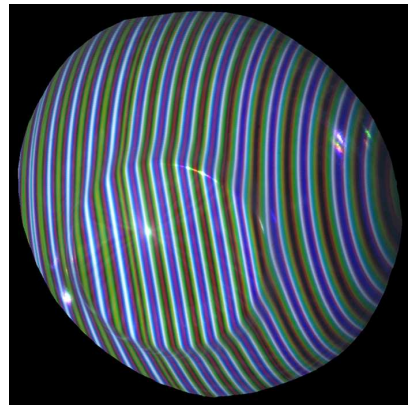

(k-m) Input images for concave bowl using color Logical XOR codes

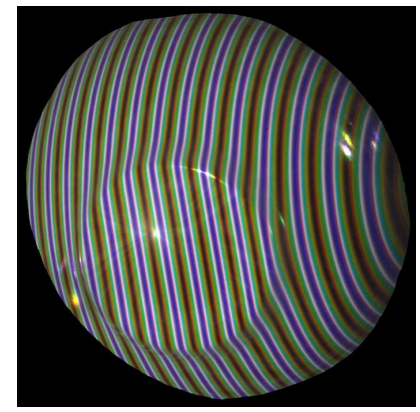

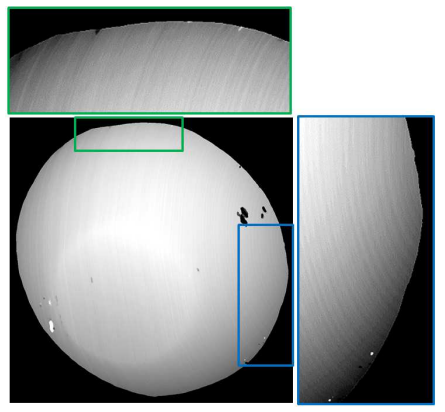

(n) Depth map

Fig. 5 Color Gray codes vs. color Logical XOR codes (color online). (a-c) Color Gray codes for a projector with 512 columns. Since 8 distinct color values are used, only 3 patterns are required to encode 512 distinct columns (binary coding requires 9 patterns). (d-f) Input images of a concave bowl for projected patterns (a-c). (g) Computed depth map. Interreflections result in erroneous reconstruction near the periphery of the bowl. Please zoom in for details. (h-j) Color Logical XOR codes, constructed by performing logical XOR operations on the color Gray codes. All the patterns have high spatial frequencies. (k-m) Input images of a concave bowl for projected patterns (h-j). (n) Computed depth map. Errors due to interreflections have been significantly mitigated. Most of the residual errors are due to pixel saturation. 
roneous reconstruction near the periphery of the bowl.

In order to construct color logical XOR codes, we start with color Gray codes. It has been shown that by performing a color calibration between projector and camera [5], the color transfer matrix between the projector and the camera can be made a diagonal matrix, and each color channel can be treated independently. With this observation, the color logical XOR codes can be constructed in a similar way as binary codes. First, a base plane is chosen. In our experiments, we chose the highest frequency pattern as the base plane. The remaining color XOR codes are made by taking the pixel-wise logical XOR of the base plane with other patterns, for each color channel independently:

$X_{c}^{i}=G_{c}^{i} \otimes G_{c}^{1}$,

for $c=\{R, G, B\}, i=\left\{2: N_{\text {color }}\right\} . X_{c}^{i}$ is the $c^{t h}$ color channel of the $i^{\text {th }}$ pattern of the color Logical XOR codes. $G_{c}^{i}$ is the $c^{t h}$ color channel of the $i^{t h}$ pattern of the color Gray codes. $G_{c}^{1}$ is the $c^{t h}$ color channel of the base plane. The captured images are first binarized in each color channel independently ${ }^{8}$ and then combined by performing a pixel-wise logical XOR operation in each color channel. This produces the $K-$ ary (in this case, $K=8$ ) decoding as if the scene was illuminated by the original low-frequency patterns. Figure 5 (h-j) show the color Logical XOR codes constructed using the algorithm described above. All the patterns have high spatial frequencies. Figures $5(\mathrm{k}-\mathrm{n})$ show the corresponding input images of the concave bowl and the computed depth map. Errors due to interreflections have been significantly mitigated. The MATLAB code for generating the patterns and decoding the input images is provided on the project web-site [1].

4.2 Maximizing the minimum stripe-widths for short-range effects

Short-range effects can blur the high-frequency base plane of the logical XOR codes. The resulting binarization error will propagate to all the decoded patterns. In order to be resistant to local blurring, patterns with low spatial frequencies must be designed. For binary patterns, this means designing patterns with large minimum stripe-width. In general, it is not feasible to find

8 Two additional images of the scene, one under all white illumination, and one under all black illumination were acquired to establish the per-pixel intensity thresholds for binarization. such codes with a brute-force search as these codes are extremely rare $^{9}$.

Fortunately, this problem has been well studied in combinatorial mathematics. There are constructions available to generate codes with large minimum stripe-widths (min-SW). The 10-bit binary Gray code with the maximum known min-SW (8 pixels) was proposed by Goddyn et al. [12]. We call these codes the maximum minSW Gray codes. These codes are shown in Figures 6 and 7 . The algorithm to construct these codes is given in [12]. The MATLAB code to generate these codes can be downloaded from the project web-site [1].

In comparison, conventional Gray codes have a minSW of 2 pixels. For Gray codes, increasing the minimum stripe-width also serves the dual purpose of reducing the maximum stripe-width. Thus, maximum min-SW Gray codes have a maximum stripe width of 32 pixels. Consequently, these codes, while being resistant to short-range effects, are also more resistant to long-range effects as compared to the conventional Gray codes. Figure 6 shows a scene consisting of industrial parts. A pico-projector was used to illuminate the scene. Due to defocus, the high-frequency patterns in the conventional Gray codes can not be decoded reliably, resulting in a loss of depth resolution. In contrast, depth map computed using maximum min-SW Gray codes does not suffer from loss of depth resolution.

\section{Ensemble of codes for general scenes}

So far, we have designed codes optimized for long or short-range effects. In general, it is not straight-forward to identify which code to use without knowing the dominant error-inducing mode of indirect illumination. This, in turn, requires a priori knowledge about scene. Moreover, indirect illumination in most real world scenes is not limited to either short or long-range effects. Codes optimized for long-range effects would make errors in the presence of short-range effects, and vice versa. In this section, we address the question: how can we handle general real world scenes that have both short and long-range indirect illumination effects?

\subsection{Depth recovery algorithm using ensemble of codes}

We show that by projecting a small ensemble of codes optimized for different effects, it is possible to handle a

\footnotetext{
9 It is relatively easy to generate codes with small maximum stripe-width. For example, we could find 10-bit codes with a maximum stripe-width of 9 pixels by performing a brute-force search. In comparison, conventional Gray codes have a maximum stripewidth of 512 pixels.
} 


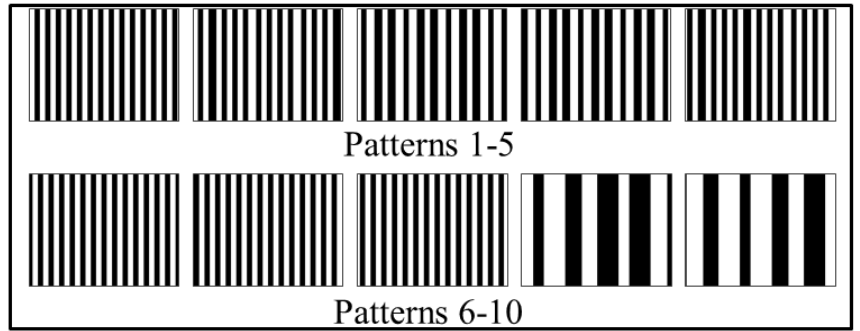

(a) Maximum min-SW Gray codes

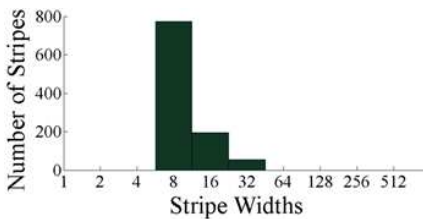

(c) Histogram of stripe-widths Maximum min-SW Gray codes

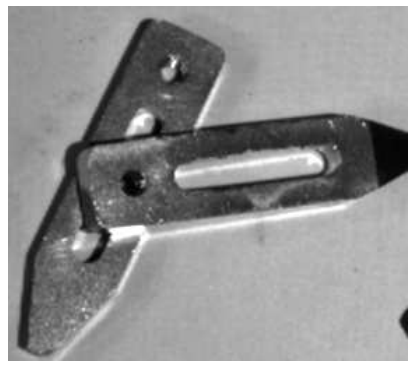

(g) Scene consisting of industrial parts

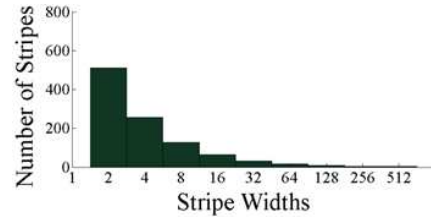

(d) Histogram of stripe-widths Conventional Gray codes

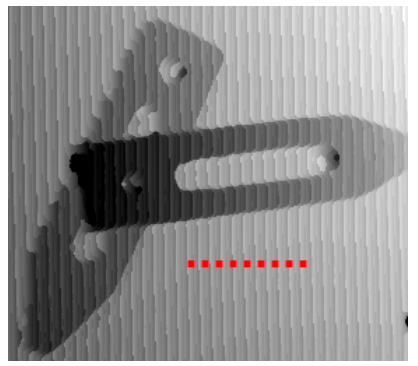

(h) Depth map using conventional Gray codes

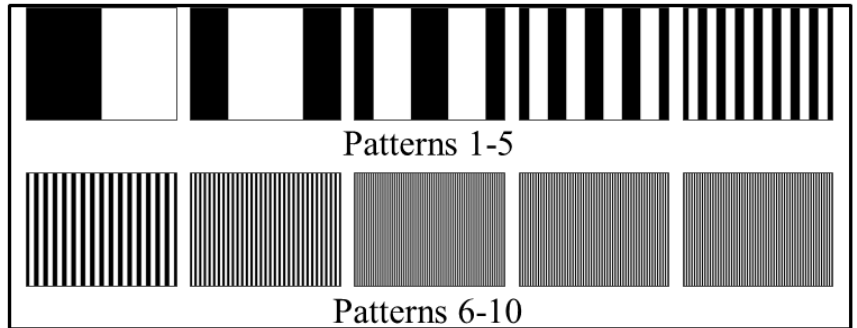

(b) Conventional Gray codes

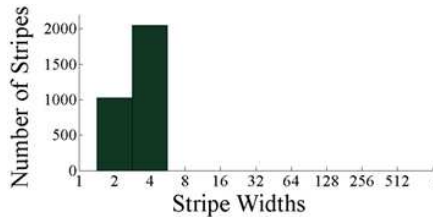

(e) Histogram of stripe-widths Logical XOR-04 codes

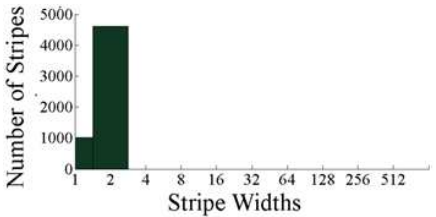

(f) Histogram of stripe-widths Logical XOR-02 codes

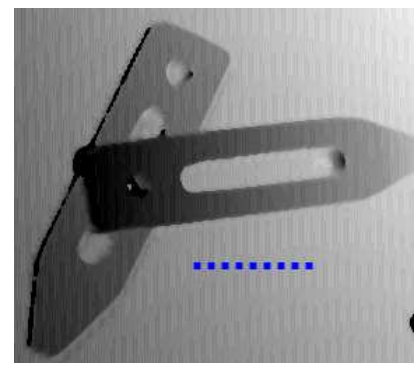

(i) Depth map using maximum min-SW Gray codes

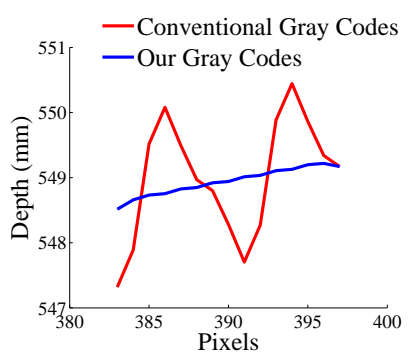

(j) Depth map comparison

Fig. 6 Designing patterns for short-range effects (color online): (a) Short-range effects such as subsurface scattering and defocus result in blurring of projected patterns. For such effects, patterns with low spatial frequencies (large stripe-widths) must be designed. We used tools from combinatorial mathematics literature to design binary patterns which maximize the minimum stripe width. These patterns are called maximum min-SW Gray codes. (b) Conventional Gray codes. (c-d) Histograms of stripe-widths for different 10-bit codes. For the maximum min-SW Gray codes, all the stripes have widths in the range [8, 32] pixels. The range of stripe-widths for conventional Gray codes, $[2,512]$ pixels, is significantly larger. For XOR-04 and XOR-02 codes, the ranges are $[2$, $4]$ and $[1,2]$ pixels respectively. (g) A scene consisting of industrial parts. (h) Due to defocus, the high-frequency patterns in the conventional Gray codes can not be decoded reliably, resulting in a loss of depth resolution. Notice the quantization artifacts. (i) Depth map computed using Gray codes with large minimum stripe-width (min-SW) does not suffer from loss of depth resolution.

large class of scenes, without a priori knowledge about scene properties. The key idea is that errors made by different codes are nearly random. Thus, if the depth values computed using two different codes is the same, with a very high probability, it must be the correct value. Based on this observation, we propose a simple depth recovery algorithm.

We project four different codes: two optimized for long-range effects (the XOR-04 and the XOR-02 codes), and two codes for short-range effects (the Gray codes with maximum min-SW and the conventional Gray codes) Each code returns a depth map of the scene, as shown in Figure 8 (a-d). The final depth value is computed by performing a simple consistency check across the depth values computed using the individual codes. If any two depth values are within a small threshold, that value is returned ${ }^{10}$.

Intuitively, the two long-range codes produce the correct depth value in the presence of long-range effects, and the short-range codes produce the correct value in the presence of short-range effects. Since there are two codes each for long and short-range effects, the consistency check will pick the correct depth value. Note that

\footnotetext{
10 Due to imperfect projector optics, insufficient camera/projector resolution or misalignment between projector and camera pixels, the depth results from individual codes might suffer from spatial aliasing. This problem is more pronounced for the high-frequency XOR codes. To prevent aliasing from affecting the final depth estimate, we apply a median filter (typically $3 \times 3$ or $5 \times 5$ ) to the individual correspondence maps before performing the consistency check.
} 


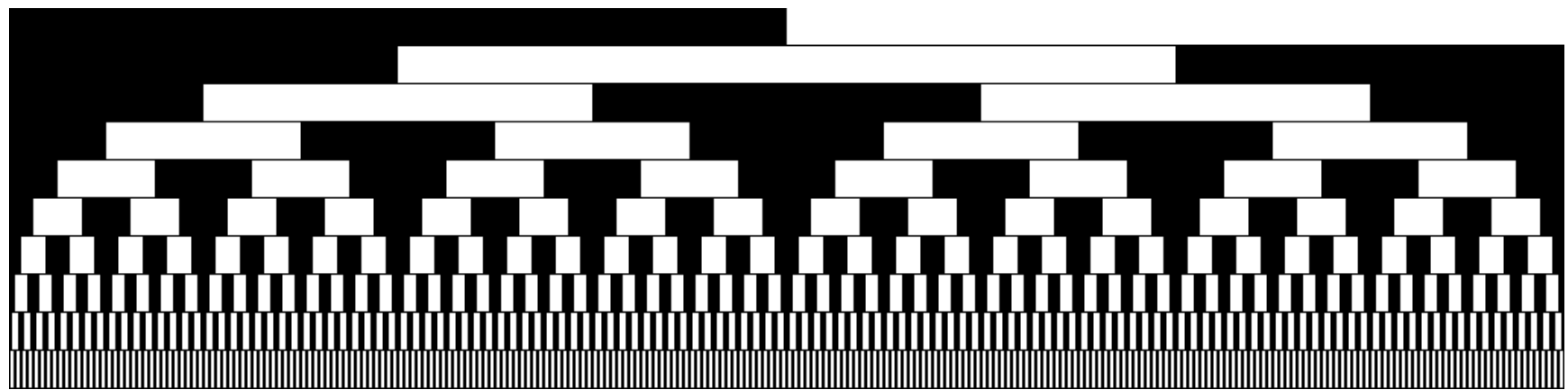

Conventional Gray Codes

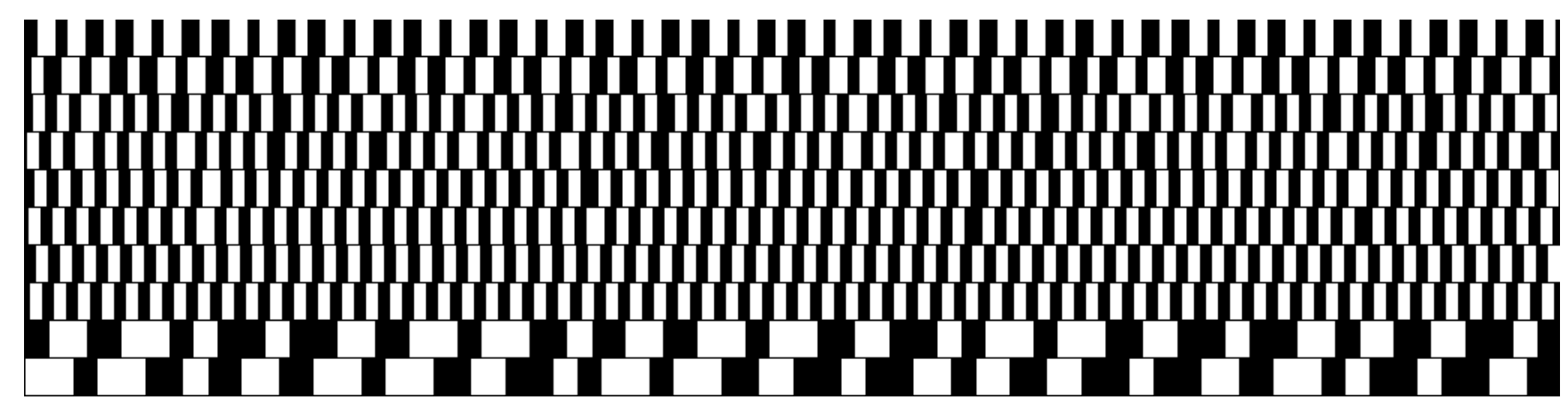

Maximum Min-SW Gray Codes

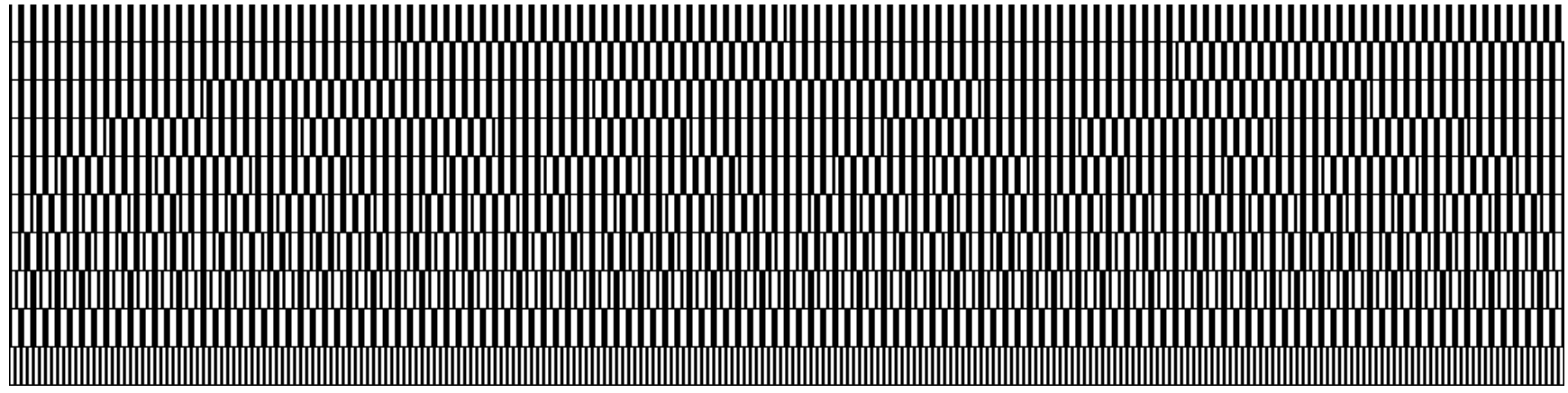

Logical XOR-04 Codes

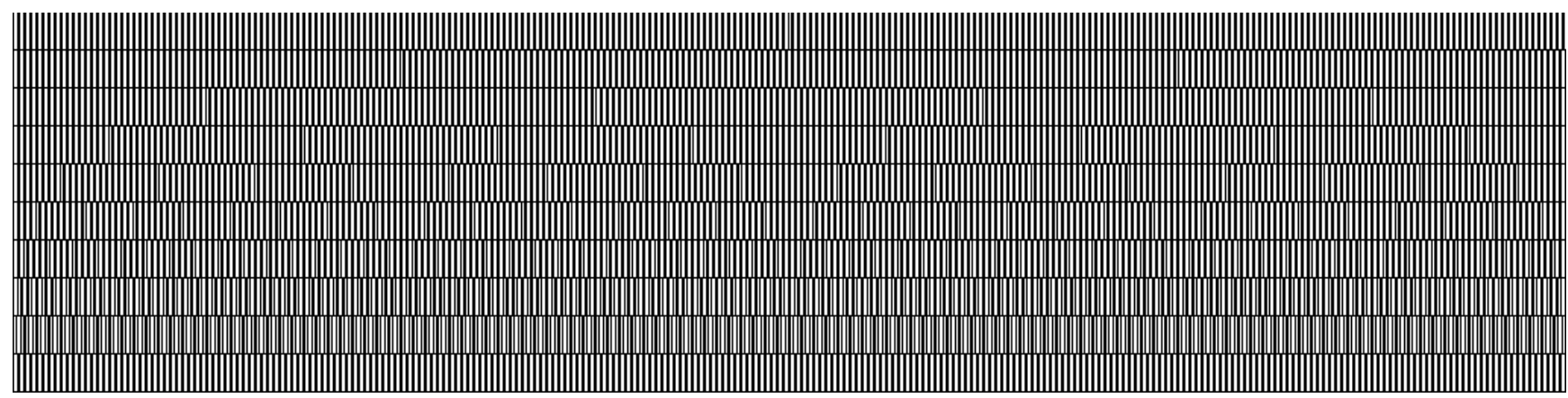

Logical XOR-02 Codes

Fig. 7 Visualization of different binary coding schemes: The patterns are for a projector with resolution $768 \times 1024$; thus, each scheme has 10 patterns. For each scheme, each row in the figure represents one pattern. Conventional Gray codes have a wide range of stripe-widths - $[2,512]$ pixels. The range for maximum min-SW Gray codes is $[8,32]$ pixels. For logical XOR-04 and XOR-02 codes, the ranges are $[2,4]$ and $[1,2]$ pixels respectively. Horizontal lines in the second row (maximum min-SW Gray codes) are actually parallel; they appear sloped because of an optical illusion called the cafe wall illusion [2]. Patterns are available at the project web-site [1].

the conventional Gray codes may lose depth resolution due to defocus of subsurface scattering. Therefore, if only the two Gray codes agree, we return the depth value computed by the maximum min-SW Gray codes. 
The pseudo-code for the method is given in Algorithm 1. MATLAB code can be downloaded from the project web-page [1].

Figure 8 (e) shows the depth map computed using the above algorithm. While the individual codes produce significant errors, the final depth map is nearly error-free. The 3D reconstruction of the scene is shown in Figure 1.

In the following, we show that the probability of two different codes making the same error, i.e., two different codes producing the same incorrect depth value, is very low. Readers not interested in the error analysis can skip Section 5.2 and go directly to results in Section 6 .

\subsection{Error analysis of the code ensemble algorithm}

Assume, without loss of generality, that the intensity coding is along the $\mathrm{x}$-dimension of the projector image plane, i.e., vertical stripes are projected. Therefore, each projector column has a unique code. For binary patterns, the code is binary. If the total number of projector columns is $M$, the code has $N$ bits, where $N=\left\lceil\log _{2}(M)\right\rceil . N$ binary patterns are projected on the scene and the camera captures $N$ images, one for each projected pattern.

Let the binary code for projector column $a$ be $C_{a}^{S}$. $S$ denotes the coding scheme, where, $S \in\{C G, M M-$ $S W, X O R 02, X O R 04\}$, corresponding to conventional Gray, maximum min-SW Gray, logical XOR02 and logical XOR04 codes, respectively. Suppose a pixel in column $a$ directly illuminates the camera pixel $x$. Let the vector of intensity values at $x$ be $I_{x}^{S}$. For correct correspondence to be established, the code $C_{a}^{S}$ should be recovered from $I_{x}^{S}$. However, various factors such as sensor noise, or illumination fluctuations or defocus and indirect illumination can result in some of the bits flipping from 0 to 1 or vice versa. This results in a decoding error. Let the recovered code be $C_{b}^{S}$. We assume that flipping of each bit in the code is independent of other bits. Then, the probability of code $C_{a}^{S}$ getting decoded incorrectly as $C_{b}^{S}$ is

$\operatorname{Pr}\left[C_{a}^{S} \rightarrow C_{b}^{S}\right]=p^{d}$,

where $p$ is the probability of one bit flipping and $d$ is the hamming distance between $C_{a}^{S}$ and $C_{b}^{S}, 0 \leq d \leq N$. $p$ is a function of sensor noise, illumination levels, scene albedos and light transport in the scene. A small value of $p$ implies that reliable decoding. A large value of $p$ indicates unreliable decoding. If we pose the problem of structured light as a communication problem, $p$ would
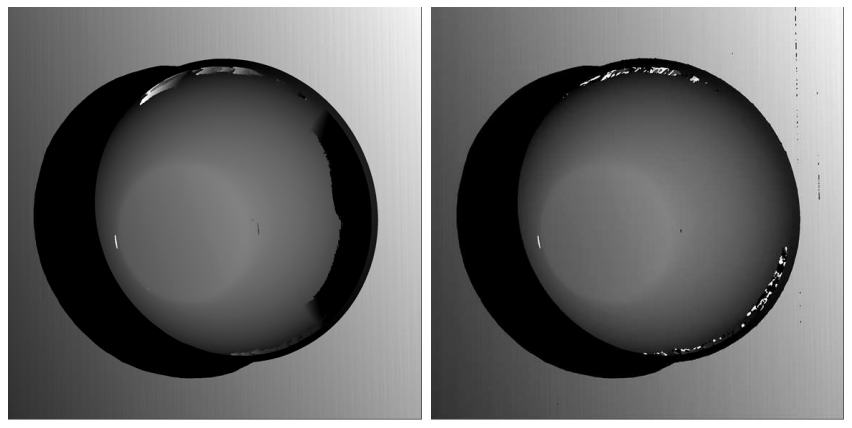

(a) Conventional Gray codes

(b) Maximum min-SW Gray codes

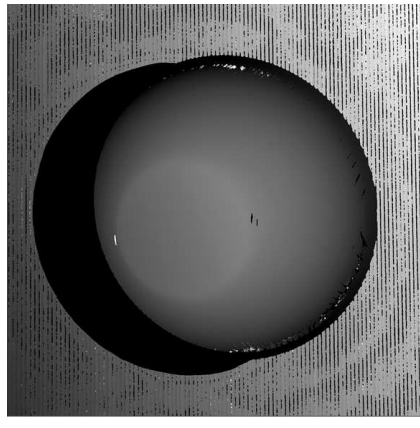

(c) XOR-04 codes

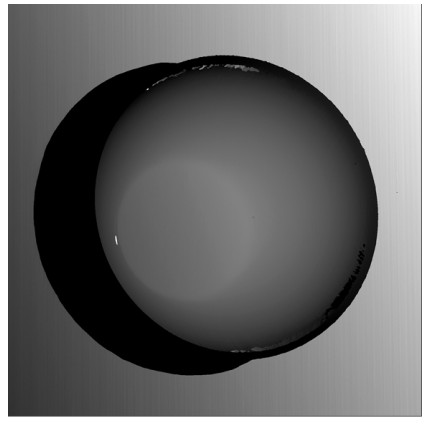

(e) Code ensemble algorithm

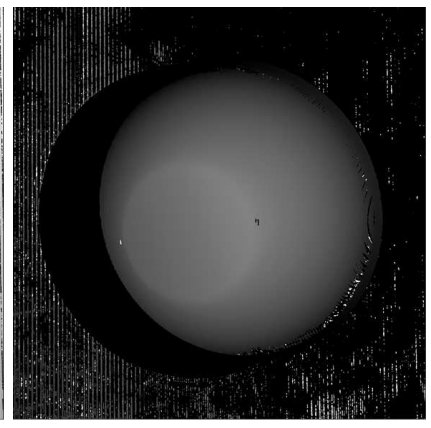

(d) XOR-02 codes

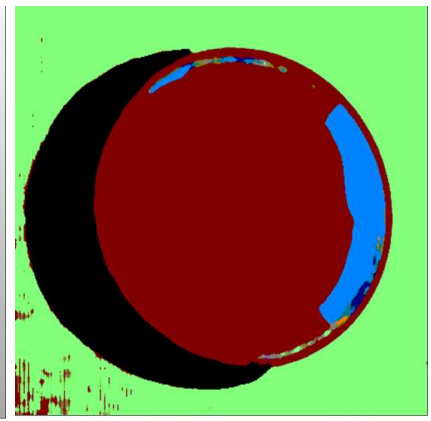

(f) Qualitative light transport
Fig. 8 Code ensemble algorithm to reconstruct scenes with multiple indirect illumination effects (color online): The scene is the same as shown in Figure 1. We project four different codes - two logical XOR codes and the two Gray codes. (a-d) Depth estimates using individual codes have errors. (e) The code ensemble algorithm performs a simple consistency check to compute a depth map with significantly fewer errors. (f) By analyzing the errors made by the individual codes, we can infer qualitative information about light-transport. Points marked in green correspond to translucent materials. Points marked in lightblue receive strong interreflections.

denote the reliability of the communication channel between the projector and the camera.

We have assumed $p$ to be constant for all bit positions. In general, since the errors due to indirect illumination are structured, $p$ is different for different bit positions. For example, for conventional Gray codes, in the presence of interreflections, since low-frequency patterns (higher significance bits) are more likely to be 

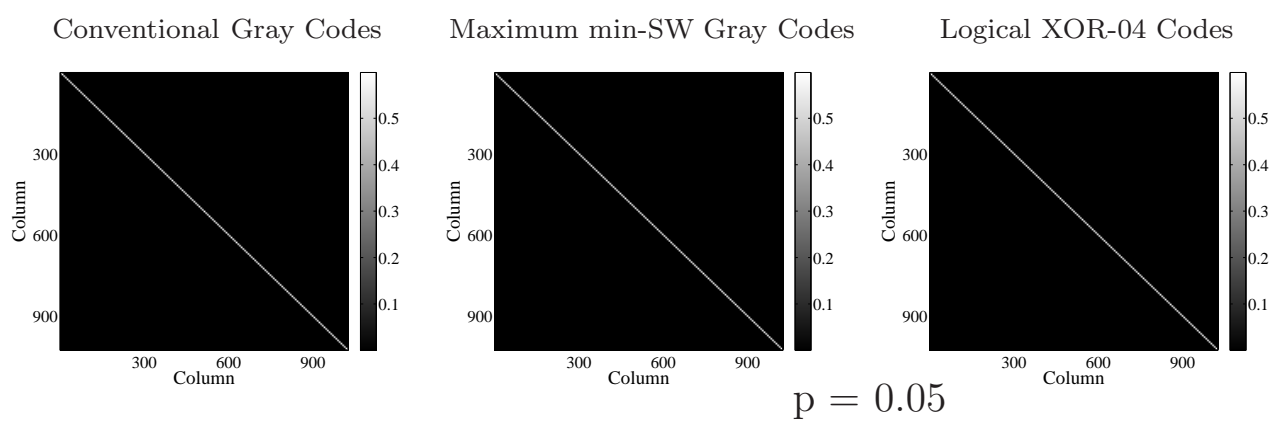

Logical XOR-02 Codes

Conventional Gray Codes
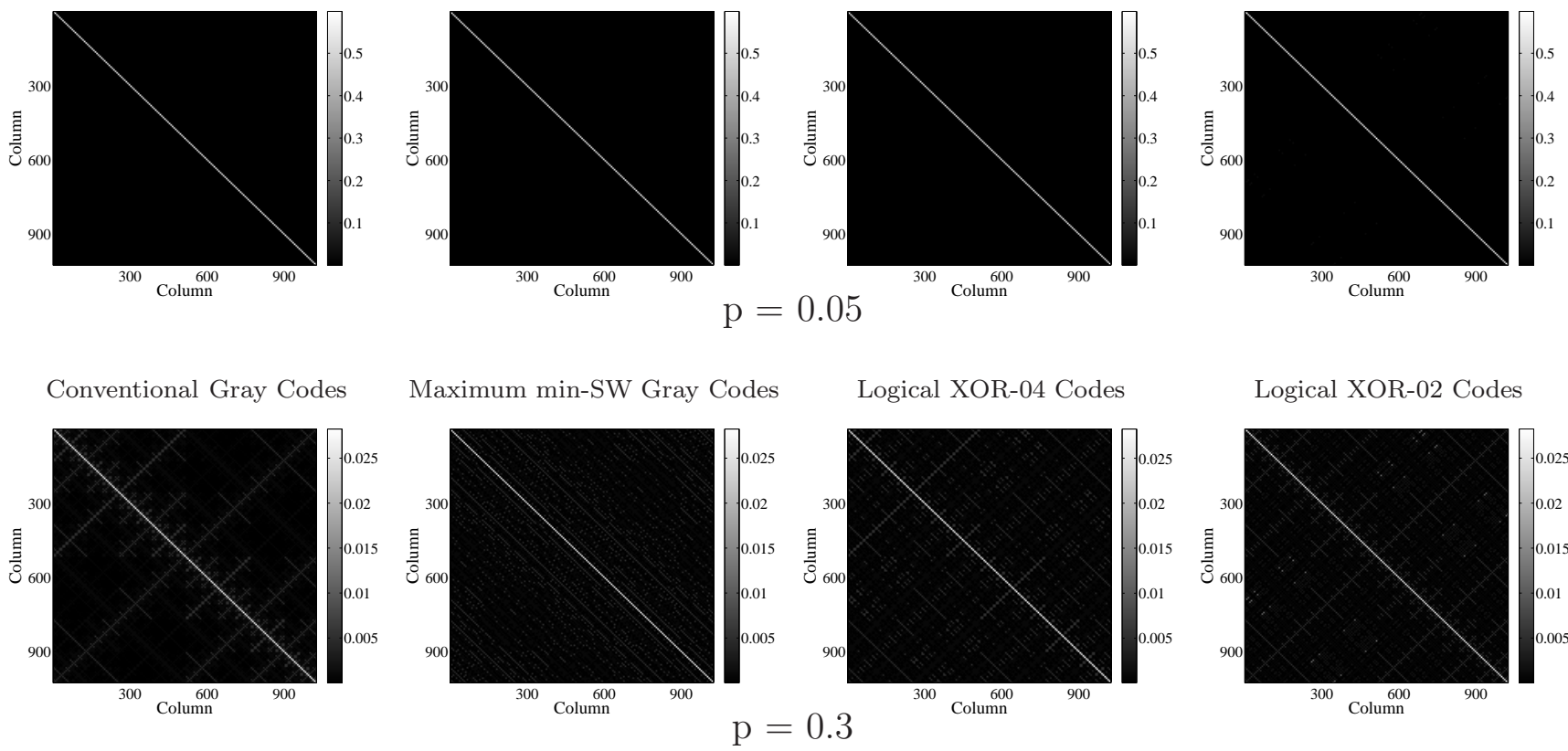

Logical XOR-02 Codes

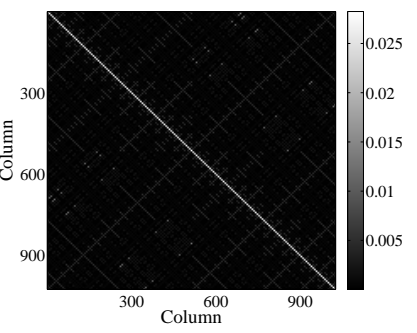

Fig. 9 Confusion matrices for different coding schemes. Confusion matrix for a coding scheme gives the probabilities of a projector column being decoded incorrectly as another projector column. For the scheme to be the most error resistant, the confusion matrix should be a diagonal matrix. We use the confusion matrices of individual coding schemes to perform error analysis of our code ensemble algorithm (Section 5). Top row: Confusion matrices for $p=0.05$, where $p$ is the probability of a single binary bit (of the $N$ bit code) flipping. $p$ is a function of the noise of the imaging and illumination system, scene albedos and light transport in the scene. For a low value of $p$, the confusion matrices for all the schemes are nearly diagonal. Bottom row: Confusion matrices for $p=0.3$. Because of a high value of $p$, the off-diagonal terms are comparable to the diagonal terms.

decoded incorrectly, $p$ is more for higher significance bits than lower significance bits. Computing the $p$ values for different codes would require knowing the scene structure a priori. One possibility is to simulate structured light decoding by rendering several scenes with global illumination. While such an approach can provide estimates of the value of $p$, it is beyond the scope of this paper. The goal of our analysis is to show that the probability of two different coding schemes making the same error is very low, for a wide range of values of $p$. If different $p$ values are estimated for different bit positions, a similar analysis can be done.

We define the confusion matrix $M^{S}$ for a coding scheme $S$ as $M^{S}(a, b)=\operatorname{Pr}\left[C_{a}^{S} \rightarrow C_{b}^{S}\right]$, where $a$ and $b$ are two projector columns. $M^{S}(a, b)$ is the probability of $C_{a}^{S}$ being decoded incorrectly as $C_{b}^{S}$. This matrix is a measure of error resilience of a given coding scheme. In order to be the most error resistant, the confusion matrix should be a diagonal matrix. Note that the confusion matrix is a function of $p$, the probability of a single bit-flip.

Figure 9 shows the confusion matrices for four coding schemes, for two different values of $p$. As expected, for a low value of $p$, the matrix is nearly diagonal for all the schemes. However, for a large value of $p$, the off-diagonal terms are comparable to the near-diagonal terms. This can result in large decoding errors. Note that the structure of the confusion matrices for the logical XOR codes is similar to the conventional Gray codes as the former are derived from the latter.

The code ensemble algorithm (Section 5.1) produces an error if the same decoding error happens for two different schemes. For the camera pixel $x$, suppose the correct corresponding projector column is $a$. The joint probability of the column $a$ being incorrectly decoded as the column $b$, for two different coding schemes $S 1$ and $S 2$ is

$$
\begin{aligned}
& \operatorname{Pr}\left[\left(C_{a}^{S 1} \rightarrow C_{b}^{S 1}\right) \&\left(C_{a}^{S 2} \rightarrow C_{b}^{S 2}\right)\right]= \\
& \operatorname{Pr}\left[C_{a}^{S 1} \rightarrow C_{b}^{S 1}\right] . \operatorname{Pr}\left[C_{a}^{S 2} \rightarrow C_{b}^{S 2}\right] .
\end{aligned}
$$

This follows from the independence of the decoding process for the two schemes. These probabilities form the joint error probability matrix $P^{(S 1, S 2)}$, where $P^{(S 1, S 2)}(a, b)=M^{S 1}(a, b) \times M^{S 2}(a, b)$. Figure 10 shows the matrices for 6 pairs of schemes. The off-diagonal values are small. Finally, we note that a column $a$ can be incorrectly decoded as any other column $b$. So, the probability that the code ensemble algorithm will result 

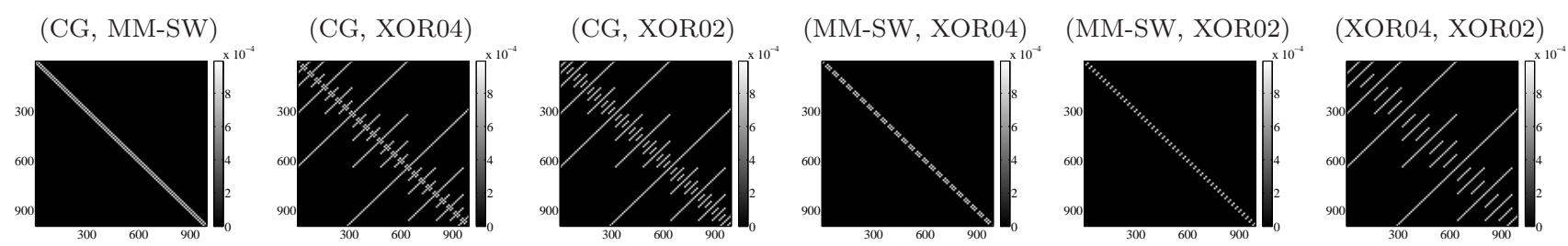

Joint error probability matrices for different pairs of coding schemes $(\mathbf{p}=\mathbf{0 . 0 5})$
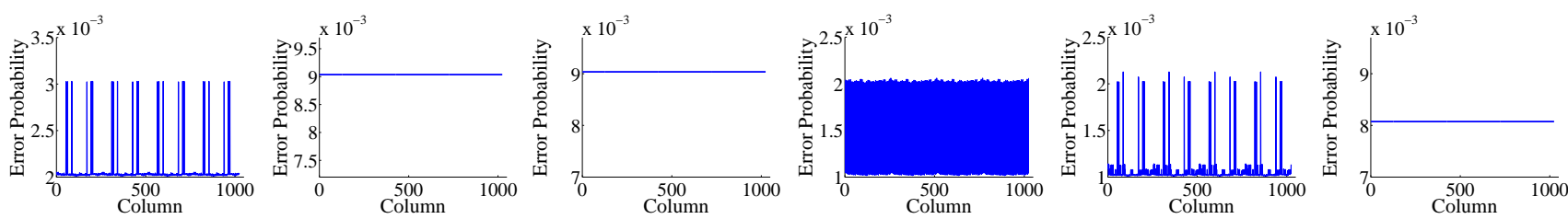

Error probabilities for our code ensemble algorithm $(p=0.05)$
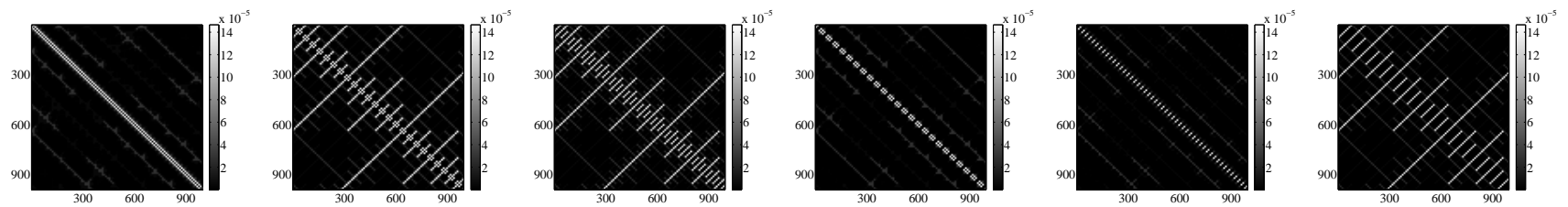

Joint error probability matrices for different pairs of coding schemes $(\mathbf{p}=\mathbf{0 . 3})$
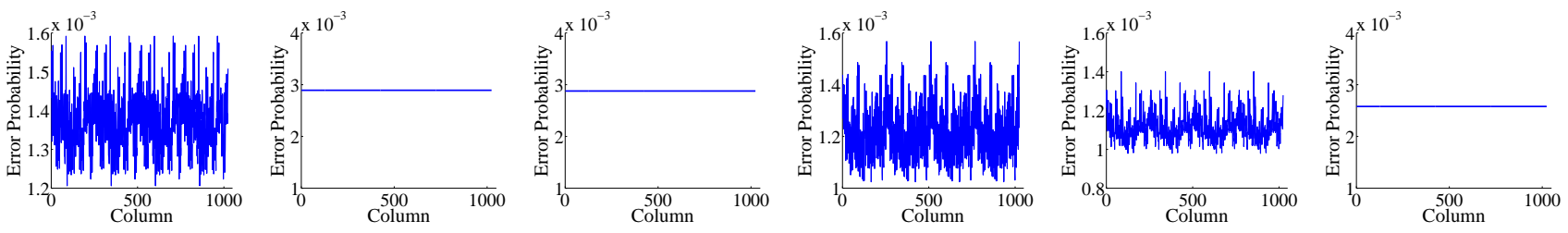

Error probabilities for the code ensemble algorithm $(\mathrm{p}=\mathbf{0 . 3})$

Fig. 10 Error analysis of our code ensemble algorithm (Section 5.1). First and third rows: Joint error probability matrices for different pairs of schemes, for different values of $p$. Most of the off-diagonal values are of the order of $10^{-6}$. Second and fourth rows: Sum of rows of the joint error probability matrices. The resulting plots are the probabilities that the code ensemble algorithm will result in a decoding error, for each projector column. Most of the probability values are less than $1 \%$.

\begin{tabular}{|c|c|c|c|c|c|c|}
\hline & (CG, MM-SW) & (CG, XOR04) & (CG, XOR02) & (MM-SW, XOR04) & (MM-SW, XOR02) & (XOR04, XOR02) \\
\hline $\mathrm{p}=0.05$ & $0.2 \%$ & $0.9 \%$ & $0.9 \%$ & $0.2 \%$ & $0.1 \%$ & $0.2 \%$ \\
$\mathrm{p}=0.1$ & $0.3 \%$ & $1.4 \%$ & $1.4 \%$ & $0.3 \%$ & $0.1 \%$ & $1.2 \%$ \\
$\mathrm{p}=0.3$ & $1.4 \%$ & $0.3 \%$ & $0.3 \%$ & $0.1 \%$ & $0.1 \%$ & $0.3 \%$ \\
$\mathrm{p}=0.5$ & $0.1 \%$ & $0.1 \%$ & $0.1 \%$ & $0.1 \%$ & $0.1 \%$ & $0.1 \%$ \\
\hline
\end{tabular}

Fig. 11 Mean error probabilities for the code ensemble algorithm. This table gives the mean probabilities of a pair of schemes making the same decoding error. These are computed by taking the mean of the error probabilities for all the projector columns (Figure 10, second and fourth rows). Most of the values are less than 1\%, with the maximum being $1.4 \%$.

\begin{tabular}{|c|c|c|c|c|c|c|}
\hline & (CG, MM-SW $)$ & (CG, XOR04) & (CG, XOR02) & (MM-SW, XOR04) & (MM-SW, XOR02) & (XOR04, XOR02) \\
\hline $\mathrm{p}=0.05$ & 0.01 & 1.03 & 1.03 & 0.02 & 0.02 & 1.06 \\
$\mathrm{p}=0.1$ & 0.06 & 1.67 & 1.67 & 0.07 & 0.07 & 1.74 \\
$\mathrm{p}=0.3$ & 0.25 & 0.52 & 0.51 & 0.29 & 0.28 & 0.56 \\
$\mathrm{p}=0.5$ & 0.33 & 0.33 & 0.33 & 0.33 & 0.33 & 0.33 \\
\hline
\end{tabular}

Fig. 12 Mean decoding error (in pixels) for the code ensemble algorithm. A decoding error results in an incorrect depth estimate. The magnitude of the depth error is directly proportional to the column error $|a-b|$, where $a$ is the correct column number and $b$ is the decoded (incorrect) column number. This table gives the mean column decoding errors for different pairs of schemes. Most of the errors are less than 1 pixel, with the maximum being 1.67 pixels. 
in a decoding error for the column $a$ is the sum of the $a^{\text {th }}$ row of the matrix $P^{(S 1, S 2)}$

$$
P^{(S 1, S 2)}(a)=\sum_{b} P^{(S 1, S 2)}(a, b) .
$$

Figure 10 (second and fourth rows) shows the plots for $P^{(S 1, S 2)}(a)$ with respect to $a$ for different pairs of schemes. Note that most of the probability values are less than $1 \%$. Figure 11 shows the mean probability of error for different pairs of schemes, where the mean is taken over all the projector columns. Most of the values are less than $1 \%$, with the maximum being $1.4 \%$.

Mean depth error: A decoding error results in an incorrect depth estimate. The magnitude of the depth error is directly proportional to the column error $\mid a-$ $b \mid$, where $a$ is the correct column number and $b$ is the decoded column number. The expected column error $E^{(S 1, S 2)}$ for a pair of schemes $S 1$ and $S 2$ is

$$
E^{(S 1, S 2)}=\frac{1}{M} \sum_{a, b}|a-b| P^{(S 1, S 2)}(a, b)
$$

where $M$ is the total number of projector columns. Figure 12 shows the mean column decoding error for different pairs of schemes, under different noise levels. Most of the errors are less than 1 pixel, with the maximum being 1.67 pixels. While this analysis was done for a projector with 1024 columns, it can be extended in a similar way for a different number of columns.

\section{Experiments and Results}

In our experiments, for phase-shifting, we project 18 patterns ( 3 frequencies, 6 shifts for each frequency). For modulated phase-shifting [8], we project 162 patterns (9 modulated patterns for each phase-shifting pattern). For our ensemble codes, we project a total of 42 patterns - 10 patterns for each of the 4 codes, 1 all-white pattern and 1 all-black patterns. Images captured under the all-white and all-black illumination patterns are used to establish per-pixel intensity thresholds for binarization.

Scenes with subsurface scattering and defocus: Figure 6 shows a scene consisting of industrial parts. Due to defocus, the high-frequency patterns in the conventional Gray codes are not decoded reliably, resulting in a loss of depth resolution. Depth map computed using maximum min-SW Gray codes does not suffer from loss of depth resolution. Figure 13 and 14 shows objects and scenes with strong subsurface scattering.
Translucent materials are often characterized by low direct component. Since modulated phase shifting [8] relies on explicitly separating the direct and the indirect components, it suffers from low signal-to-noise-ratio for highly translucent materials. The resulting depth maps are severely degraded due to noisy. Our code ensemble does not rely on explicit direct-indirect separation, resulting in significantly better reconstructions.

Scenes with diffusion: Next, we consider scenes which have only long-range effects. Figures 15 and 16 show scenes comprising of thin, nearly transparent surfaces. In both cases, light diffuses through the material and is reflected from the background/interior, creating longrange optical interactions. Consequently, conventional Gray codes and phase-shifting result in large errors in the reconstructed shape. For some moderately difficult scenes, such as the shower curtain in Figure 15, it is sufficient to use only one of our codes, instead of the full ensemble.

Scenes with multiple indirect illumination effects: Next, we show scenes which have multiple indirect illumination effects (but each scene point receives either long or short-range effects). Figures 1 and 8 show a scene consisting of a bowl on a marble slab. Depth estimates using individual codes (Figures 8(a-d)) have errors due to various indirect illumination effects. The depth estimate using our code ensemble has significantly fewer errors. Corresponding 3D reconstructions are shown in Figure 1. By analyzing the errors made by the individual codes, qualitative information about light-transport can be inferred, as shown in Figure 8 (f). Points marked in green correspond to translucent materials. Points marked in light-blue receive strong interreflections.

The scenes in Figures 17 and 18 have both interreflections and subsurface scattering. Modulated phaseshifting performs poorly on translucent materials, whereas conventional Gray codes and phase-shifting produce errors due to interreflections. In contrast, reconstruction produced using our ensemble of codes has significantly reduced errors.

Finally, we consider scenes which have points that receive both short and long-range effects. Figure 19 shows results for a cup made of styrofoam. Since styrofoam is weakly translucent, points inside the cup receive both subsurface scattering and strong interreflections. Conventional Gray codes produce large errors in the recovered shape. The spatial frequencies of the $\max$ min-SW Gray codes are not sufficiently high to prevent errors. However, accurate shape is recovered using the code ensemble because of high-frequency XOR-02 and XOR-04 codes. Figure 20 shows shape recovery re- 


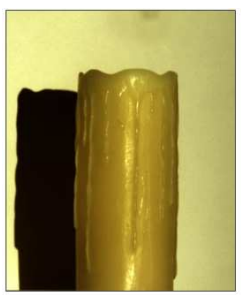

(a) Object

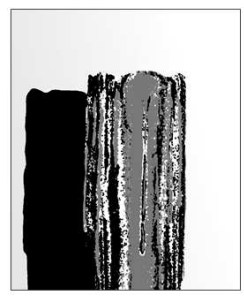

(b) Modulated PS

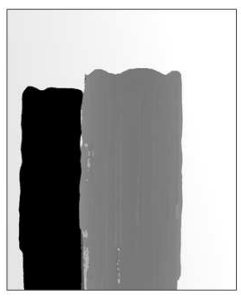

(c) Code ensemble

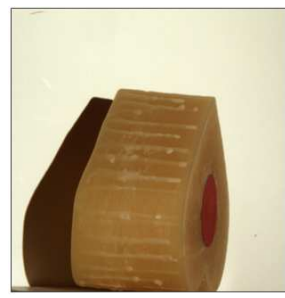

(d) Object

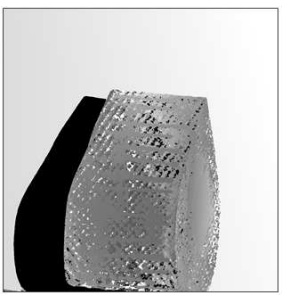

(e) Modulated PS

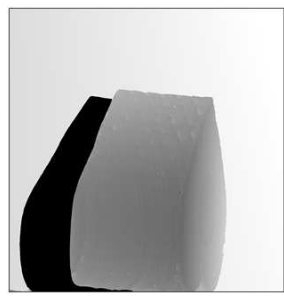

(f) Code ensemble

Fig. 13 Reconstructing translucent wax objects: Translucent materials (a,d) are often characterized by low direct component. Since modulated phase shifting relies on explicitly separating the direct and the indirect components, it suffers from low signal-tonoise-ratio for highly translucent materials. The resulting depth maps are very noisy (b,e). Our code ensemble does not rely on explicit direct-indirect separation, resulting in better quality depth-maps $(\mathrm{c}, \mathrm{f})$.

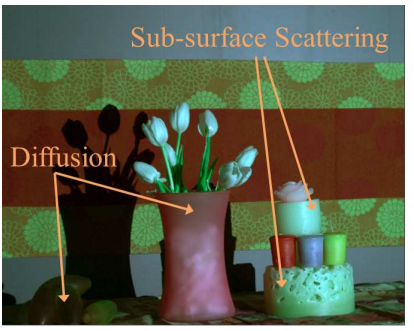

(a) Candles and flower vase

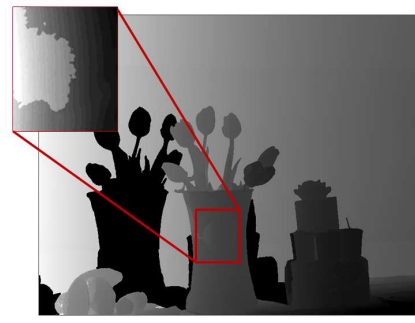

(b) Conventional Gray codes

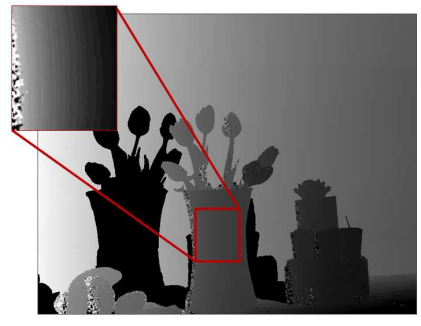

(c) Modulated phase shifting

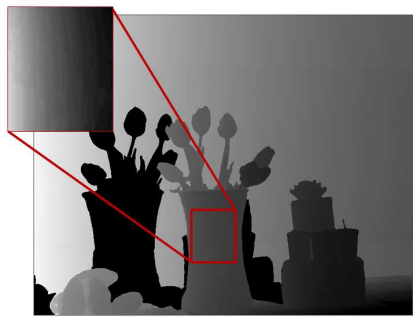

(d) Our code ensemble

Fig. 14 Candles and flower-vase: (a) The scene consists of a flower vase and some wax candles. The flower vase is made of diffuse glass, resulting in diffusion of light. The wax candles have subsurface scattering. Depth from phase shifting (b) has errors on the flower vase, while modulated phase shifting results in errors on the candles (c). Depth map using our code ensemble (d) is nearly error free. Zoom in for details.

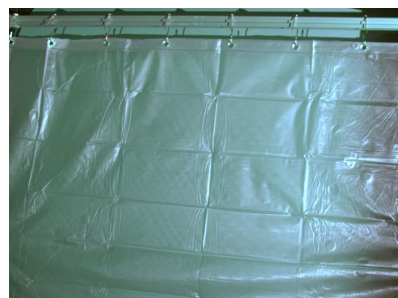

(a) Shower curtain

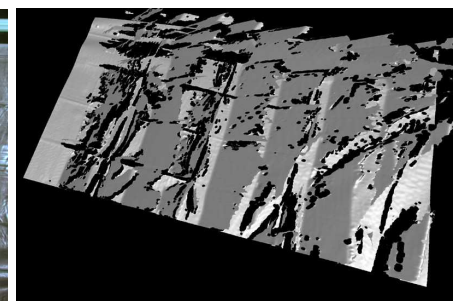

(b) Conventional Gray codes

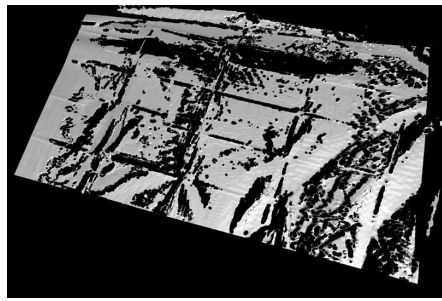

(c) Phase shifting

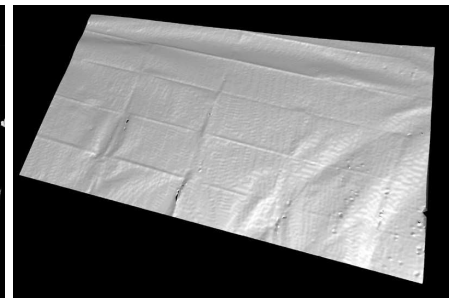

(d) The XOR-04 codes

Fig. 15 Shower-curtain: (a) Light diffuses through the curtain and is reflected from the background, creating long-range optical interactions. Consequently, (b) conventional Gray codes and (c) phase-shifting result in large holes in the estimated shape. The correct shape of the curtain is approximately planar, with small ripples. (d) Reconstruction using our logical XOR-04 codes is nearly error free, with the same number of input images as the conventional Gray codes.

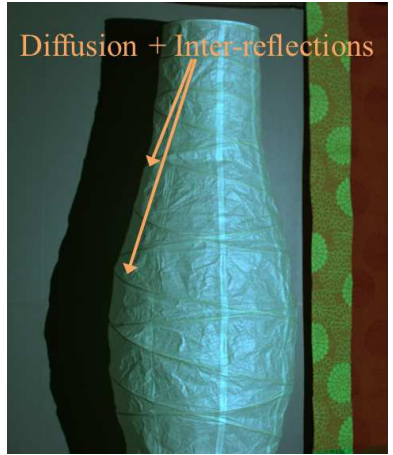

(a) Ikea lamp

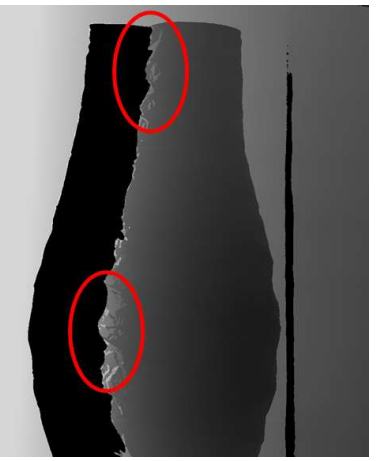

(b) Conventional Gray codes

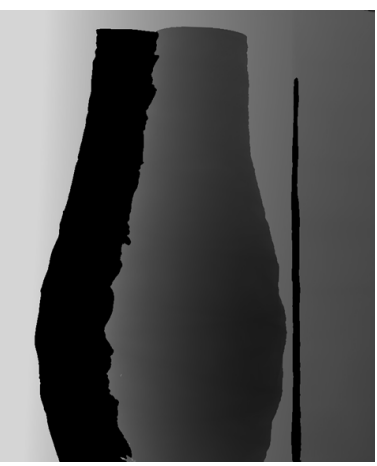

(c) Our code ensemble

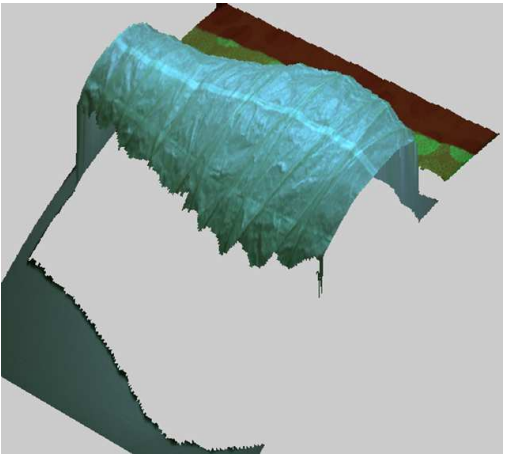

(d) Visualization of (c)

Fig. 16 Reconstructing an Ikea lamp (color online): The lamp is made of thin translucent paper. Light diffuses inside the lamp, bounces inside and comes back out. (b) Conventional Gray codes result in errors near the periphery of the lamp (marked in red). (c) Depth map using our code ensemble. (d) 3D visualization of (c). 

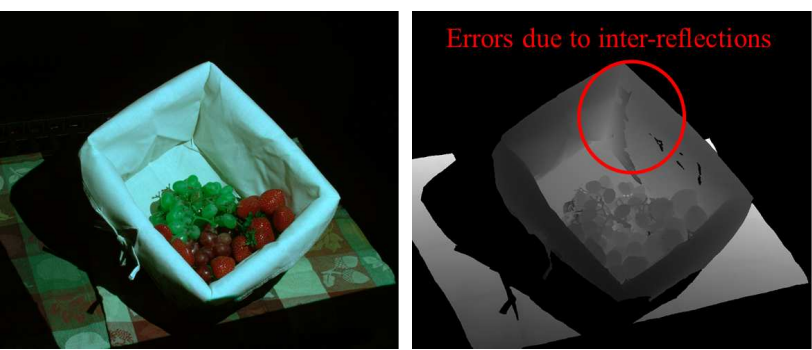

(a) Fruit-basket

(b) Conventional Gray (11 images)

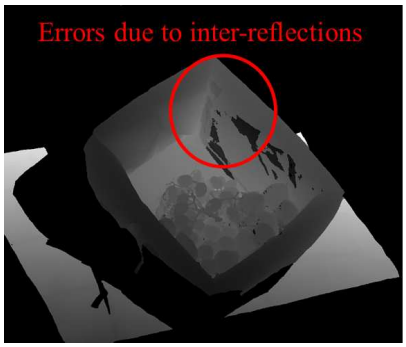

(c) Phase shifting (18 images)

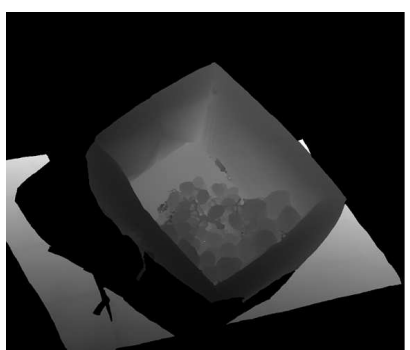

(e) Code ensemble (42 images)

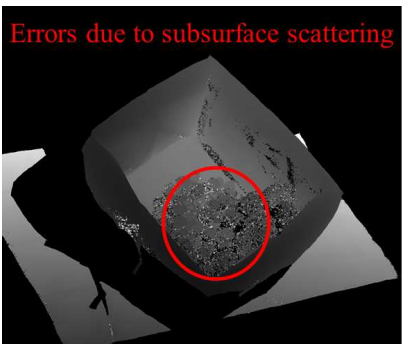

(d) Modulated phase shifting [8] (162 images)

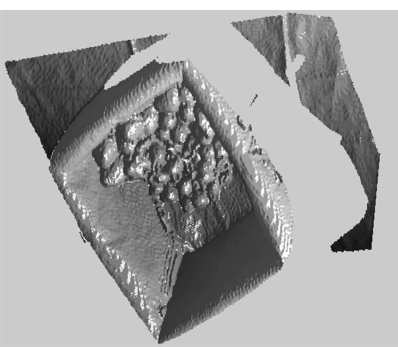

(f) 3D Visualization for (e)

Fig. 17 Measuring 3D shape of a fruit-basket (color online). (a) This scene has both interreflections (corner of the fruitbasket) and subsurface scattering on the fruits. (b-c) Conventional Gray codes and phase-shifting produce erroneous depthmaps to interreflections (errors marked in red). (d) Modulated phase shifting produces errors on the translucent fruits due to low direct component. (e) Our technique using an ensemble of codes results in significantly fewer errors. Parentheses contain the number of input images.

sults for a wax bowl. Points inside the bowl receive strong subsurface scattering. Since the interreflections are weak (the bowl is shallow), the code ensemble produces an accurate shape. For more results and highresolution images, see the project web-page [1].

Figure 21 shows a failure case - a deep container made of highly translucent wax. Points inside the container receive both strong interreflections and strong subsurface scattering. Since none of the four codes compute the correct shape, the code ensemble fails to reconstruct the object.

Comparisons with Couture et al. [9]: Recently, Couture et al. [9] proposed an approach to deal with interreflections by projecting a large number (200) of
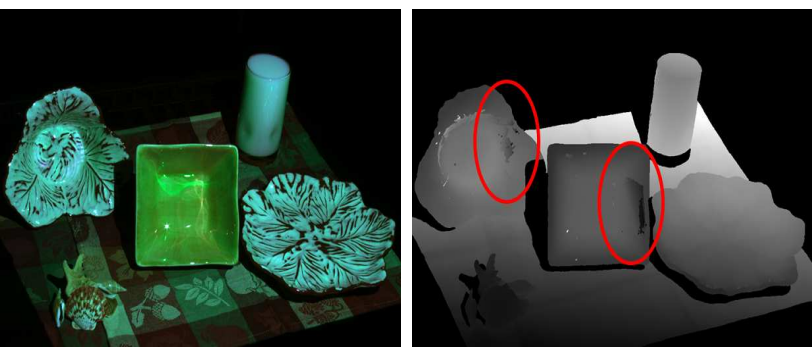

(a) Bowls and milk

(b) Conventional Gray (11 images)
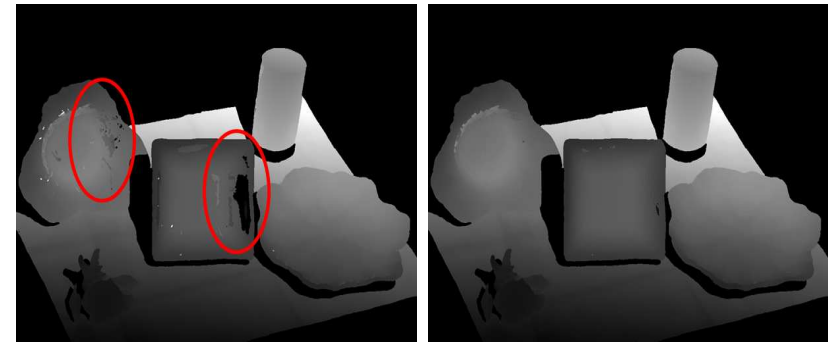

(c) Phase-shifting (18 images)

(d) Code ensemble (42 images)
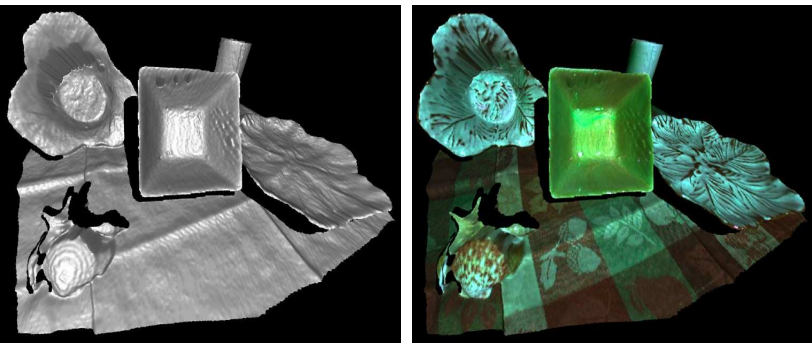

3D Visualizations for $(d)$

Fig. 18 Depth map computation for the bowls and milk scene (color online). (b) Conventional Gray codes and (c) phase-shifting result in errors (marked in red) at points receiving strong interreflections. (d) Depth-map using our code ensemble.

random high-frequency patterns on the scene. Figure 22 shows comparisons of their approach with our XOR-04 codes, which have similar spatial frequencies as used in [9]. Since all three scenes have strong interreflections, the conventional Gray codes result in large errors. The random high-frequency codes successfully remove the errors. The XOR-04 codes produce results of the same accuracy, while requiring an order of magnitude fewer images (12 versus 200).

\section{Error detection and correction}

The patterns presented in the previous section can successfully prevent a large fraction of errors. For highly challenging scenes, however, some errors might still remain. An example is shown in Figure 23. This object is a concave lamp made of brushed metal. This is a challenging object due to strong, high-frequency interreflections. Figure 24 (e) shows the reconstruction re- 


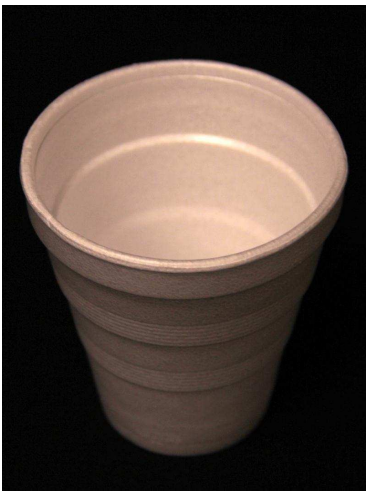

(a) Styrofoam cup

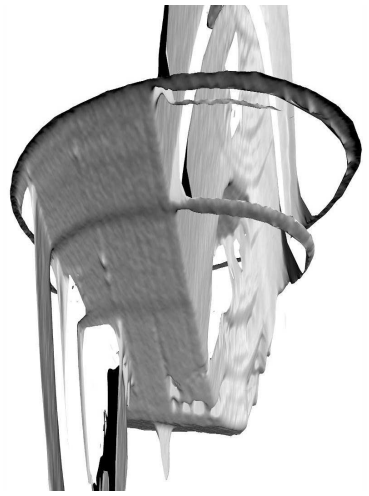

(b) Conventional Gray codes

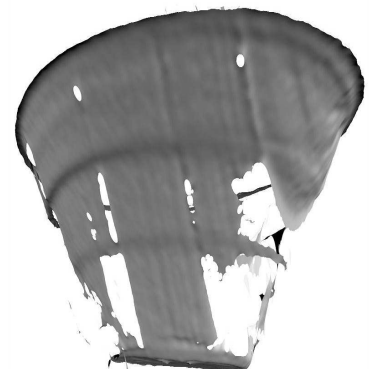

(c) Max min-SW Gray codes

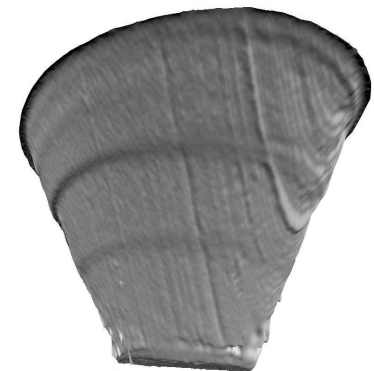

(d) Code ensemble

Fig. 19 Styrofoam cup (interreflections+subsurface scattering): Styrofoam is weakly translucent. Points inside the cup receive both subsurface scattering and strong interreflections. (b) Conventional Gray codes produce large errors in the recovered shape. (c) The spatial frequencies of our max min-SW Gray codes are not sufficiently high to prevent errors. (d) Accurate shape is recovered using our code ensemble because of high-frequency XOR-02 and XOR-04 codes.

sults using the code ensemble. While the reconstruction is better as compared to individual codes, a significant amount of errors remain. For building a reliable shape measurement system, it is critical to detect and correct these residual errors.

Traditionally, error detection and correction strategies from communication theory have been adopted in the context of structured light. An example is the Hamming error correcting codes used by Minou et al. [25]. These techniques treat structured light coding-decoding as a signal transmission problem. Although good for handling random sensor/illumination noise, these codes can not handle the systematic errors made due to indirect illumination. In this section, we present strategies for detecting and correcting such errors.

\subsection{Error detection}

Our error detection algorithm is based on a simple observation. The consistency check proposed in the previous section, in addition to preventing errors, can also be used for detecting errors. For a pixel, if none of the four codes agree, it is marked as an error pixel, as illustrated in Figure 24 (f). It is possible that one of the four values might be the correct value. However, as there is an error correction stage to follow, we take a conservative approach and classify such pixels as error pixels. Since no extra patterns need to be projected, the error detection stage does not place any overhead in terms of acquisition time.

Park et al. [32,31] use similar consistency checks across range scans acquired from different view points. By registering different scans and comparing the values from different scans, they remove spurious measure-

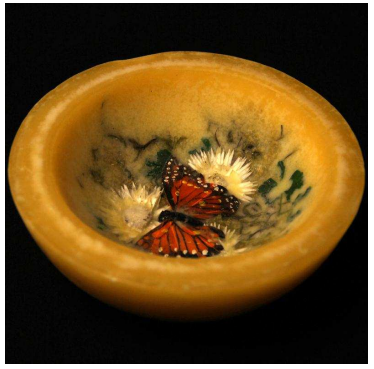

(a) Wax bowl

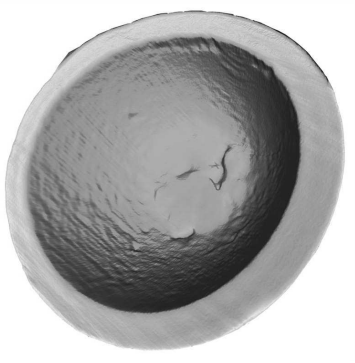

(b) Code ensemble
Fig. 20 Wax bowl (interreflections+subsurface scattering): (a) Points inside the bowl receive weak interreflections (the bowl is shallow) and strong subsurface scattering. (b) Shape computed using the code ensemble algorithm.

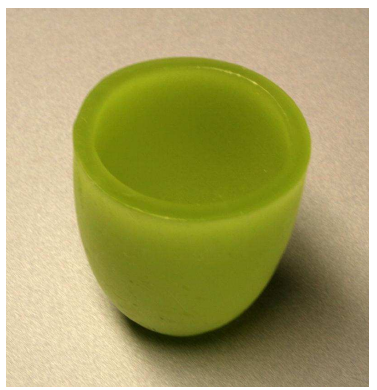

(a) Deep wax container

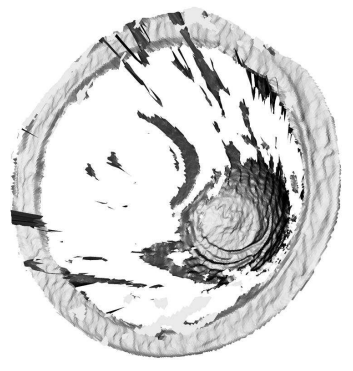

(b) Code ensemble
Fig. 21 Deep wax container (failure case): Points inside the container receive both strong interreflections and strong subsurface scattering. Since none of the four codes compute the correct shape, the code ensemble fails to reconstruct the object.

ments due to specular interreflections. In contrast, our technique does not require moving the acquisition setup or the object. 

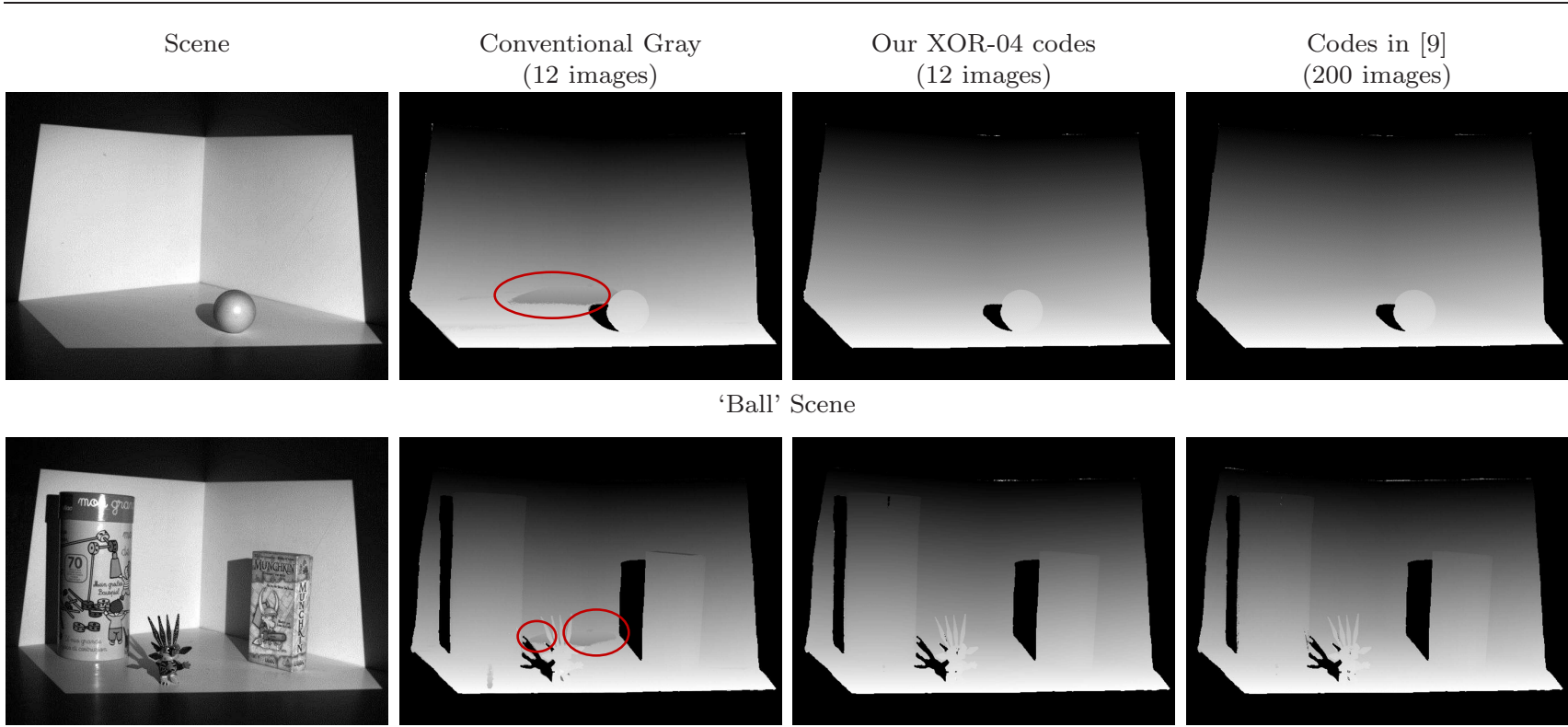

'Games' Scene
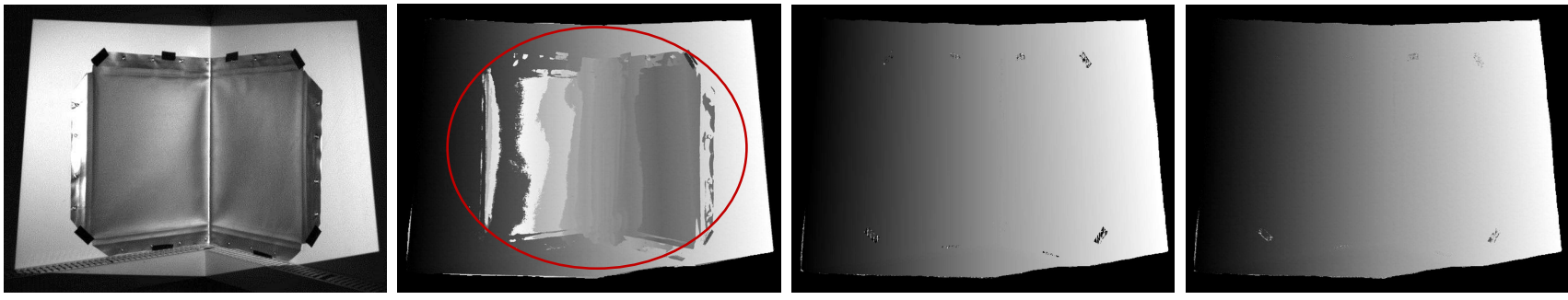

'Corner' Scene

Fig. 22 Comparisons with Couture et al. [9]: First column shows scenes with interreflections. Second column shows shape recovered with conventional Gray codes. Since all the scenes have strong interreflections, conventional Gray codes result in large errors (marked in red). The random high-frequency codes [9] successfully remove the errors, as shown in the third column. The XOR-04 codes produce results of the same accuracy (fourth column), while requiring an order of magnitude fewer images (12 versus 200).

\subsection{Error correction}

To correct the errors, we iteratively collect additional images while illuminating only the scene points corresponding to the error pixels. This technique, based on the work of $\mathrm{Xu}$ et al. [38], progressively reduces the amount of indirect illumination, resulting in reduction of the error pixels. In the subsequent iterations, the scene points which are already decoded correctly are not illuminated. This is achieved using illumination masks, as shown in Figures $24(\mathrm{~g}, \mathrm{~h})$. By progressively reducing the number of points getting illuminated (and hence, interreflections), the residual errors are reduced. By acquiring images in 2 extra iterations ${ }^{11}$, we achieve a nearly perfect reconstruction.

Conventional Gray codes can not reconstruct a large portion of the object. Modulated phase-shifting [8] can not remove the high-frequency interreflections, resulting in large errors. The mean absolute errors as com-

\footnotetext{
11 We projected only the logical codes in subsequent iterations, thus requiring 82 images in total.
}

pared to the ground truth for our result, conventional Gray codes and modulated phase-shifting are $1.2 \mathrm{~mm}$, $29.8 \mathrm{~mm}$ and $43.9 \mathrm{~mm}$ respectively (height of the lamp $=$ $250 \mathrm{~mm}$ ), respectively. The ground truth was acquired by manually binarizing the captured images.

It is important to note that for this error correction strategy to be effective, the error prevention and detection stages are critical. Since our techniques correctly reconstruct a large fraction of the scene in the first iteration itself, we require only a small number of extra iterations (typically 1-2) even for challenging scenes. In comparison, the approach presented in [38] requires a large number of iterations (10-20) and images (500800). This is because it uses conventional Gray codes, which do not prevent errors in the first place. Secondly, its error detection technique, based on direct-indirect separation, is conservative. Consequently, if the direct component is low (for example, in the presence of subsurface scattering), this technique may not converge. 
Algorithm 1 Structured Light Scanning in the Presence of Indirect Illumination

1. Project patterns and capture images for the 4 codes - two Gray codes (Conventional Gray and Gray codes with maximum min-SW), and the two logical codes (XOR02 and XOR04)

2. Compute depth values for the two Gray codes using conventional decoding and the two logical codes using the logical decoding (Section 4.1).

3. Apply a median filter (e.g., $3 \times 3$ or $5 \times 5$ ) to the individual depth values to prevent propagation of aliasing errors.

4. Compare the depth values. If any two codes are consistent, return that value as the correct depth. If the two Gray codes are consistent, return the value computed by the maximum min-SW Gray codes (Section 5).

5. Error detection: Mark the camera pixels where no two codes agree as error pixels (Section 7). An example is shows in Figure 24.

6. Error correction: Mask the patterns so that only the scene points corresponding to the error pixels are lit [38]. Repeat steps $1-5$ to progressively reduce the residual errors (Section 7, Figure 24)

\section{Discussion and Limitations}

Frequencies of the projected patterns: Our methods make the following assumptions on indirect illumination. The high-frequency codes assume that indirect illumination is locally smooth. The low-frequency codes assume that the indirect illumination is local. If both these conditions are violated simultaneously, our techniques may produce incorrect results. For example, if a scene has mirror interreflections, or if the extent of subsurface scattering is significantly larger than the minimum stripe width of max min-SW codes, our techniques may fail to reconstruct the scene accurately.

This limitation is because we have classified indirect illumination into either long or short-range. To handle general scenes with a continuous range of indirect illumination effects, patterns with a continuous set of frequencies can be used. For example, it is possible to construct different band-pass codes by doing the XOR operations. Instead of only XOR-02 and XOR04 codes, depending on the scene, XOR-08, XOR-16, XOR-32 codes can be used. Alternatively, sinusoidal patterns can be used as they provide more flexibility in controlling spatial frequencies [18]. Ultimately, there is a trade-off between acquisition speed and the range of scenes that can be handled. Four sets of patterns with extreme frequencies can be considered to be the minimal set.

What are the good spatial frequencies to use? Answering this requires a more thorough analysis of the frequencies of light transport. While such an analysis is hard for general scenes, we believe that studying the

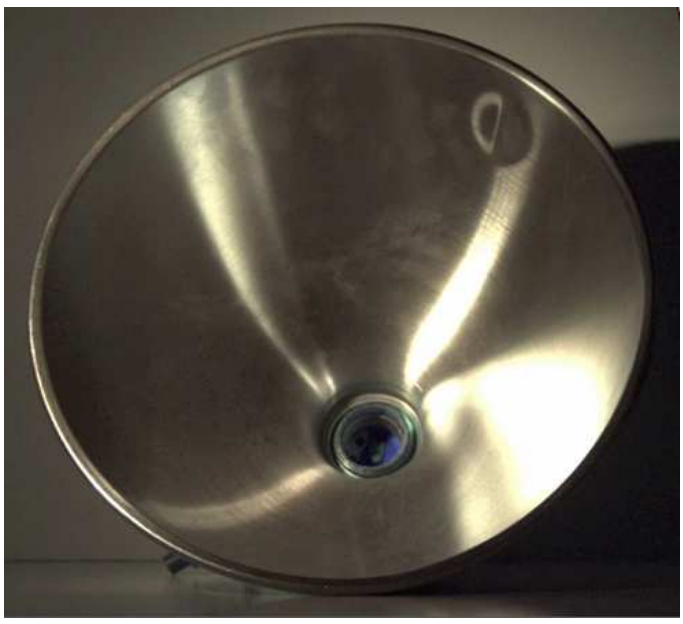

(a) Concave Metal Lamp

Fig. 23 Concave metal lamp: A highly challenging object due to strong, high-frequency interreflections.

statistics of light transport for natural scenes will provide useful insights. This forms a promising direction of future research.

Single dominant mode of indirect illumination: Our techniques assume a single dominant mode of indirect illumination for every scene point. If a scene point receives both strong short-range and long-range effects, for example, inside of a strongly translucent and deep bowl, none of the codes will produce the correct result. An example is shown in Figure 20. In this case, the code ensemble algorithm and the error correction step will not be able to retrieve the correct result. Our techniques can not handle scenes in the presence of participating media as volumetric scattering also results in both short-range and long-range interactions.

Qualitative classification of indirect illumination: The qualitative classification of indirect illumination shown in Figure 8 is specific to the projector camera configuration. So far, we haven't reached a stage where this classification can provide reliable quantitative information about the scene. For example, most of the points inside the bowl receive interreflections. But since this classification is based on the errors that the code ensemble algorithm makes, only a few points are classified as receiving interreflections.

Conventional Gray codes as short-range codes: In our code ensemble, we have considered conventional Gray codes as being resistant to short-range effects. This is an approximation. Due to local effects, the higher frequency images in the conventional Gray codes will get blurred, and might not be decoded correctly. However, since the high-frequency patterns correspond to the lower significance bits, the resulting errors are small 
Consensus-checking among the code ensemble

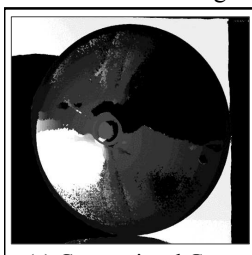

(a) Conventional Gray

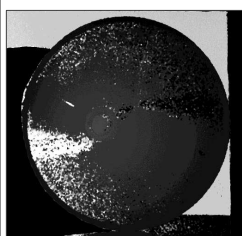

(c) Logical XOR-4

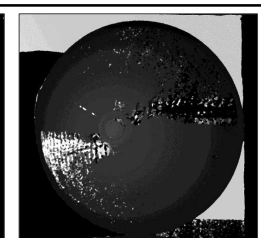

(b) Max min-SW Gray

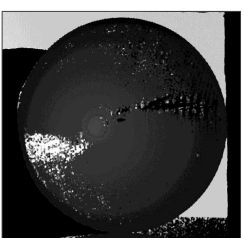

(d) Logical XOR-02
Any Two Codes Consistent

No Two Codes Consistent

Error detection using consistency check

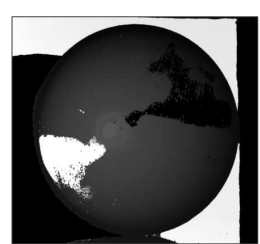

(e) Depth from Ensemble

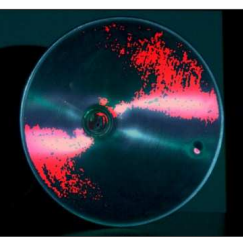

(f) Error map (Red Pixels)

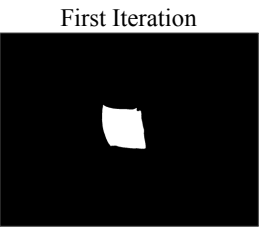

(g) Illumination Mask

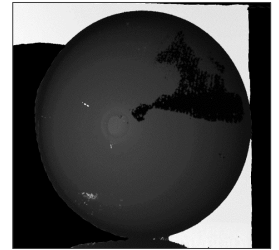

(i) Depth Map
Second Iteration

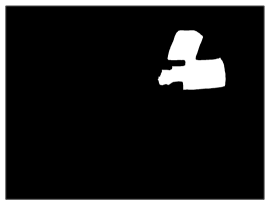

(h) Illumination Mask

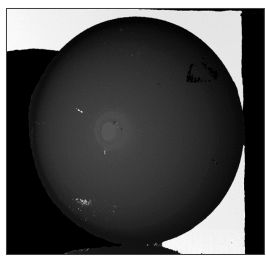

(j) Depth Map
Error correction
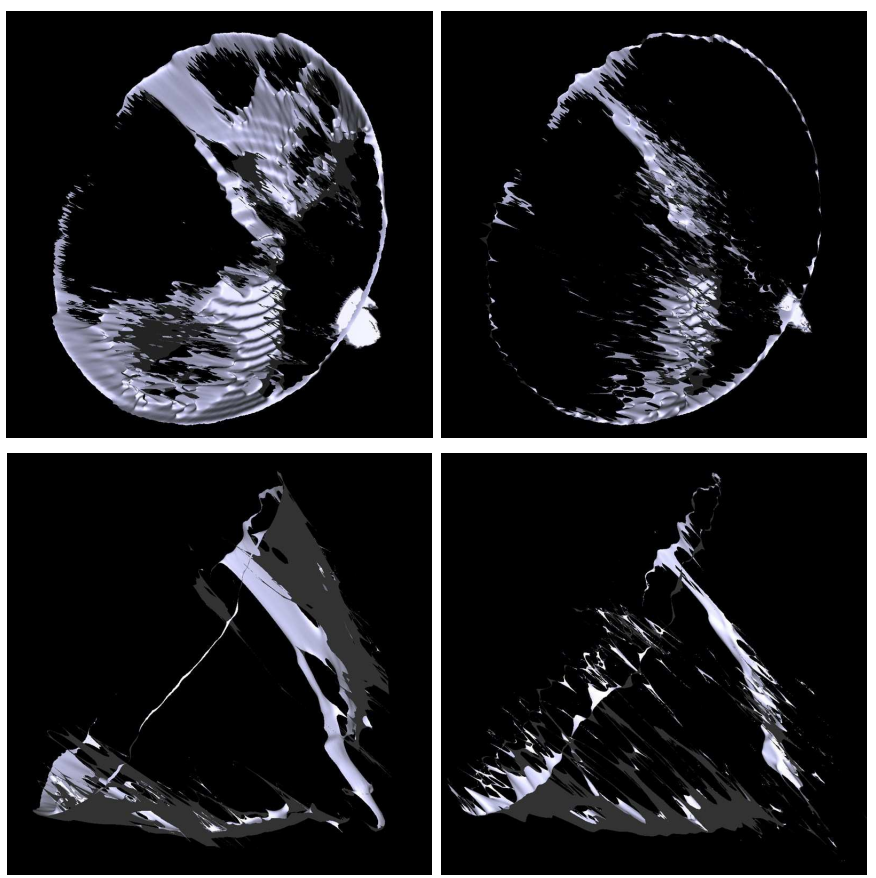

(k) Conventional Gray codes (11 images)

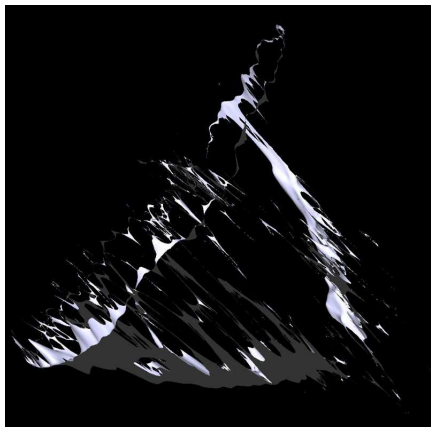

(1) Modulated phase shifting (162 images)
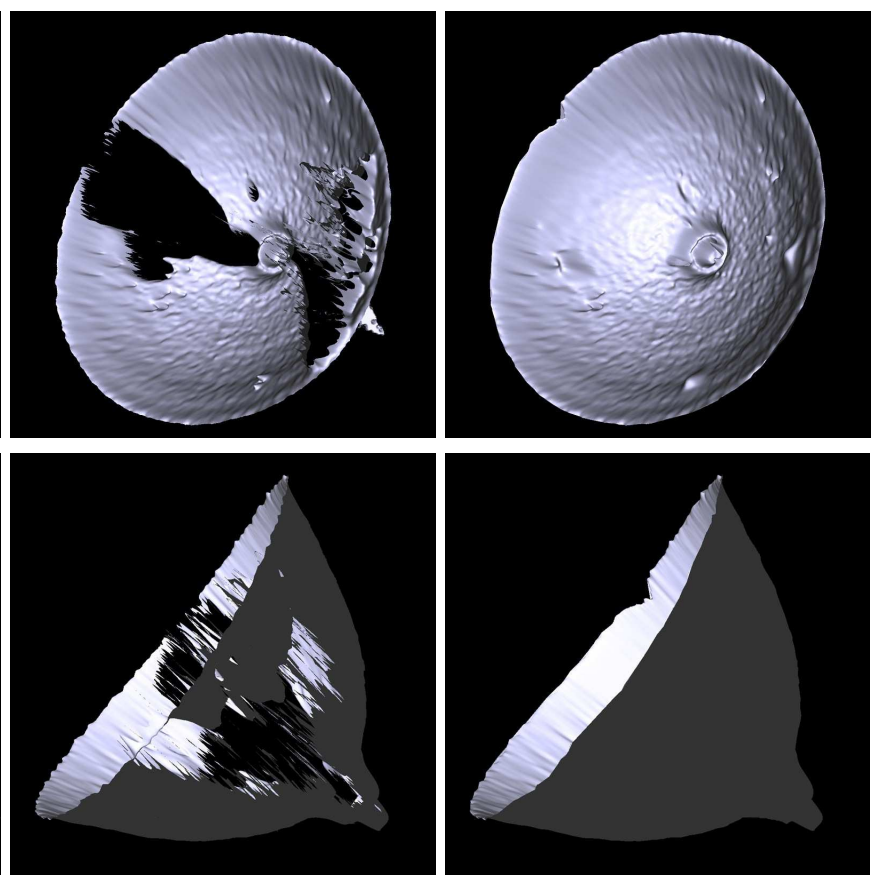

(m) Our code ensemble (42 images)

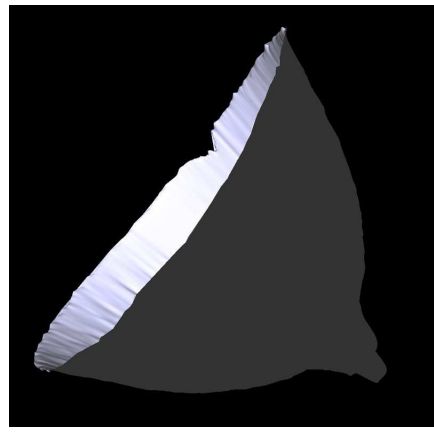

(n) After error detection and correction (82 images)

Fig. 24 Error Detection and Correction (color online): (a) We use the same consistency check as in the code ensemble algorithm for detecting errors. (a-d) Four depth maps using the individual codes. (e) Depth using the code ensemble algorithm has a significant amount of residual errors. (f) For a pixel, if no two codes agree on a depth value, it is marked as an error pixel (red). Since no extra patterns are projected, the error detection stage places no overhead in terms of acquisition time. In subsequent iterations, scene points that are already decoded correctly are not illuminated. This is achieved using an illumination masks (g,h). By progressively reducing the number of points getting illuminated (and hence, interreflections), the residual errors are reduced [38] (i,j). This object is very hard to reconstruct with existing schemes $(k, l)$. Using our techniques, we achieve a high quality reconstruction (n). The mean errors for our result (n), conventional Gray codes (k) and modulated PS (l) are $1.2 \mathrm{~mm}, 29.8 \mathrm{~mm}$ and $43.9 \mathrm{~mm}$ respectively (height of lamp $=250 \mathrm{~mm})$. The parentheses contain number of input images.

(e.g., $<4$ pixels if the last two patterns are lost). Hence, in the consistency check step, the result of conventional
Gray codes will still agree with that of the max minSW Gray codes. In this case, the value computed by the 
minimum min-SW codes is returned. A future research direction is to design more codes with large minimum stripe widths.

Acquisition speed: Our techniques are currently limited to binary codes (monochrome and color) and thus require capturing several tens of images, making them unsuitable for dynamic scenes. The number of input images can be decreased by having more than two intensity levels in the projected images. An interesting direction of future work is to extend our techniques to $N$-ary $(N>2)$ codes and continuous schemes, such as phase shifting [18], which require fewer images as compared to discrete binary patterns. The number of images can also be reduced using a priori knowledge about the scene. For example, if the scene is known to have only interreflections, then it is sufficient to use only the logical codes, e.g., XOR-04. If, however, no a priori knowledge about the scene is available, then the code ensemble should be used.

Acknowledgements. We thank Jay Thornton, Joseph Katz, John Barnwell and Haruhisa Okuda (Mitsubishi Electric Japan) for their help and support. Mohit Gupta was partially supported by ONR grant N00014-11-1-0295. Srinivasa Narasimhan was partially supported by NSF grants IIS-0964562 and CAREER IIS-0643628 and a Samsung SAIT GRO grant. Ashok Veeraraghavan was partially supported by NSF Grants IIS-1116718 and CCF-1117939. The authors thank Vincent ChapdelaineCouture for sharing their data-sets.

\section{References}

1. http://graphics.cs.cmu.edu/projects/ StructuredLight3DScanning/.

2. http://mathworld.wolfram.com/CafeWallIllusion.html.

3. D. G. Aliaga and Y. Xu. Photogeometric structured light: A self-calibrating and multi-viewpoint framework for accurate 3D modeling. In $C V P R, 2008$.

4. B. Atcheson, I. Ihrke, W. Heidrich, A. Tevs, D. Bradley, M. Magnor, and H. Seidel. Time-resolved 3D capture of nonstationary gas flows. ACM Trans. Graph., 27(3), 2008.

5. D. Caspi, N. Kiryati, and J. Shamir. Range imaging with adaptive color structured light. IEEE PAMI, 20, 1998.

6. M. K. Chandraker, F. Kahl, and D. J. Kriegman. Reflections on the generalized bas-relief ambiguity. In CVPR, 2005.

7. T. Chen, H. P. A. Lensch, C. Fuchs, and H. peter Seidel. Polarization and phase-shifting for 3D scanning of translucent objects. In $C V P R, 2007$.

8. T. Chen, H.-P. Seidel, and H. P. A. Lensch. Modulated phaseshifting for 3D scanning. In CVPR, 2008.

9. V. Couture, N. Martin, and S. Roy. Unstructured light scanning to overcome interreflections. In $I C C V, 2011$.

10. M. C. Er. On generating the $\mathrm{n}$-ary reflected gray codes. IEEE Transactions on Computers, C-33(8), 1984.

11. J. Ghring. Dense 3-D surface acquisition by structured light using off-the-shelf components. Videometrics and Optical Methods for 3D Shape Measurement, 4309, 2001.
12. L. Goddyn and P. Gvozdjak. Binary gray codes with long bit runs. The electronic journal of combinatorics, 2003.

13. G. Godin, J.-A. Beraldin, M. Rioux, M. Levoy, L. Cournoyer, and F. Blais. An assessment of laser range measurement of marble surfaces. In Proc. of Fifth Conference on optical 3D measurement techniques, 2001.

14. J. Gu, T. Kobayashi, M. Gupta, and S. K. Nayar. Multiplexed illumination for scene recovery in the presence of global illumination. In ICCV, 2011.

15. J. Gu, S. K. Nayar, E. Grinspun, P. N. Belhumeur, and R. Ramamoorthi. Compressive structured light for recovering inhomogeneous participating media. In ECCV, 2008.

16. M. Gupta, A. Agrawal, A. Veeraraghavan, and S. G. Narasimhan. Structured light 3d scanning in the presence of global illumination. In $C V P R, 2011$.

17. M. Gupta, S. G. Narasimhan, and Y. Y. Schechner. On controlling light transport in poor visibility environments. In CVPR, 2008.

18. M. Gupta and S. K. Nayar. Micro phase shifting. In $C V P R$, 2012.

19. M. Gupta, Y. Tian, S. G. Narasimhan, and L. Zhang. (De) Focusing on global light transport for active scene recovery. In $C V P R, 2009$.

20. C. Hermans, Y. Francken, T. Cuypers, and P.Bekaert. Depth from sliding projections. In $C V P R, 2009$.

21. M. Holroyd, J. Lawrence, and T. Zickler. A coaxial optical scanner for synchronous acquisition of $3 \mathrm{~d}$ geometry and surface reflectance. ACM Trans. Graph., 29(3), 2010.

22. E. Horn and N. Kiryati. Toward optimal structured light patterns. Proc. International Conference on Recent Advances in 3-D Digital Imaging and Modeling, 1997.

23. I. Ihrke, K. N. Kutulakos, H. P. A. Lensch, M. Magnor, and W. Heidrich. State of the art in transparent and specular object reconstruction. STAR Proceedings of Eurographics, 2008.

24. S. Liu, T. T. Ng, and Y. Matsushita. Shape from secondbounce of light transport. In $E C C V, 2010$.

25. M. Minou, T. Kanade, and T. Sakai. A method of time-coded parallel planes of light for depth measurement. Transactions of IECE Japan, 64(8), 1981.

26. N. J. W. Morris and K. N. Kutulakos. Reconstructing the surface of inhomogeneous transparent scenes by scatter trace photography. In $I C C V, 2007$.

27. S. G. Narasimhan, S. K. Nayar, B. Sun, and S. J. Koppal. Structured light in scattering media. In ICCV, 2005.

28. S. K. Nayar, K. Ikeuchi, and T. Kanade. Shape from Interreflections. IJCV, 6(3), 1991.

29. S. K. Nayar, G. Krishnan, M. D. Grossberg, and R. Raskar. Fast separation of direct and global components of a scene using high frequency illumination. ACM Trans. Graph., 25(3), 2006.

30. D. Nehab, S. Rusinkiewicz, J. Davis, and R. Ramamoorthi. Efficiently combining positions and normals for precise $3 \mathrm{~d}$ geometry. ACM Trans. Graph., 24(3), 2005.

31. J. Park and A. C. Kak. Multi-peak range imaging for accurate 3D reconstruction of specular objects. In $A C C V, 2004$.

32. J. Park and A. C. Kak. 3D modeling of optically challenging objects. IEEE Transactions on Visualization and Computer Graphics, 14(2), 2008.

33. J. Posdamer and M. Altschuler. Surface measurement by space-encoded projected beam systems. Computer Graphics and Image Processing, 18(1), 1982.

34. J. Salvi, S. Fernandez, T. Pribanic, and X. Llado. A state of the art in structured light patterns for surface profilometry. Pattern Recognition, 43, 2010.

35. E. Steger and K. N. Kutulakos. A theory of refractive and specular 3D shape by light-path triangulation. IJCV, 76(1), 2008. 
36. M. Trobina. Error model of a coded-light range sensor. Technical Report, 1995.

37. P. M. Will and K. S. Pennington. Grid coding: A preprocessing technique for robot and machine vision. Artificial Intelligence, 2(3-4), 1971.

38. Y. Xu and D. Aliaga. An adaptive correspondence algorithm for modeling scenes with strong interreflections. IEEE TVCG, 2009

39. L. Zhang and S. K. Nayar. Projection Defocus Analysis for Scene Capture and Image Display. ACM Trans. Graph., 25(3), 2006.

40. S. Zhang. High-resolution, three-dimensional shape measurement. Ph.D. Thesis, Stony Brook University, 2005.

41. S. Zhang, D. V. D. Weide, and J. Oliver. Superfast phaseshifting method for 3-D shape measurement. Optics Express, 18(9), 2010 